\title{
Quantitative Untersuchung der Anhangsorgane der Achselhaut bei den japanischen Kindern.
}

\author{
Von
}

\section{Masahisa Endo.}

Aus dem Anatomischen Institut der Keio Universität, Tokyo.

Inhalt.

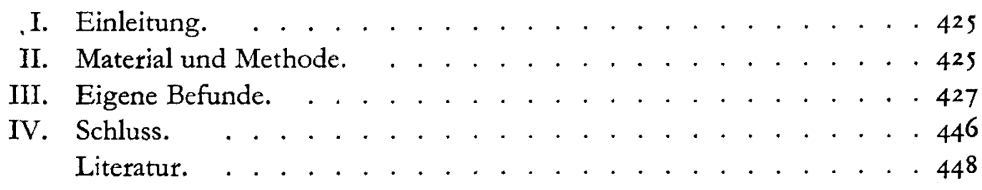

\section{Einleitung.}

Die Mengenverhältnisse der Anhangsorgane der Haut der verschiedenen Körperteile bei den verschiedenen Rassen, Altersstufen und beiden Geschlechtern haben viele Autoren unseres Institutes schon ausführlich erforscht. In der vorliegenden Arbeit habe ich die Anhangsorgane der Achselhaut, d.h. Schweissdrüse (a- und e-Drüse), Talgdrüse und Haarbalgmuskel, welche bis jetzt von niemand quantitativ verfolgt worden sind, bei den japanischen Kindern eingehend untersucht.

\section{Material und Methode.}

Die Materialien stammen aus 12 japanischen Kindern (neugeborene bis 8.5 jährige), wie in der Tabelle I gezeigt.

Das Hautstück von ca. I qcm Grösse der rechten Achselhöhle wurde nach dem Tode möglichst früh mit genügendem Unterhautfettgewebe herausgeschnitten und dann in 10\% iger wässeriger Formalinlösung fixiert. Danach wurden die Hautstücke mit möglichst ebener Oberfläche in Zelloidin eingebettet und in Flächenschnitte von $30 \mu$ Dicke serienweise zerlegt. Die Färbung geschah mit Hämatoxylin-Eosin.

Die Schweiss-, Talgdrüse und der Haarbalgmuskel in jedem Schnitt wurden in so maliger Vergrösserung auf ein Papierstück mit einem bestimm- 


\begin{tabular}{|c|c|c|c|c|c|c|c|c|c|c|c|c|c|}
\hline \multicolumn{2}{|c|}{ 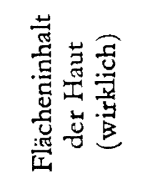 } & 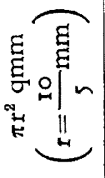 & 2 & $\hat{n}$ & $=$ & $\hat{n}$ & $\approx$ & $\hat{a}$ & $\hat{~}$ & $=$ & $\approx$ & $=$ & $=$ \\
\hline \multicolumn{2}{|c|}{ 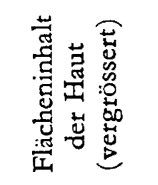 } & 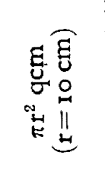 & .2 & $=$ & $=$ & $=$ & a & $\hat{\alpha}$ & $\hat{2}$ & $\therefore$ & $\therefore$ & $\therefore$ & $=$ \\
\hline \multicolumn{2}{|c|}{ 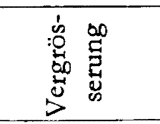 } & $\begin{array}{l}\overrightarrow{\widetilde{I J}} \\
\stackrel{0}{0} \\
0\end{array}$ & 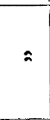 & = & 2 & 2 & $\hat{=}$ & $=$ & $=$ & a & $\therefore$ & $\hat{a}$ & $\hat{a}$ \\
\hline \multicolumn{2}{|c|}{ 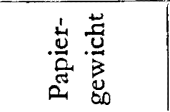 } & $\begin{array}{l}\ddot{5} \\
0 \\
\dot{0}\end{array}$ & $\hat{n}$ & $\hat{n}$ & $\hat{a}$ & $=$ & a & $=$ & $\therefore$ & 2 & 2 & $\therefore$ & $\therefore$ \\
\hline \multicolumn{2}{|c|}{ 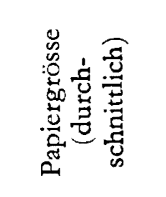 } & 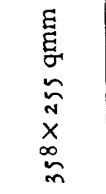 & $=$ & $=$ & $\hat{n}$ & $\hat{n}$ & $=$ & $=$ & $\therefore$ & $\therefore$ & $=$ & $=$ & $=$ \\
\hline \multirow{4}{*}{ 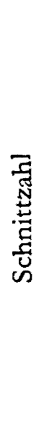 } & \multirow{2}{*}{ 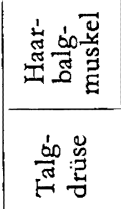 } & 1 & 웅 & $\stackrel{\infty}{\sim}$ & $\tilde{\sim}$ & $\approx$ & $\therefore$ & $\stackrel{4}{8}$ & $\hat{N}$ & ง & $\stackrel{\text { ก }}{2}$ & $\stackrel{\circ}{\circ}$ & $\stackrel{m}{m}$ \\
\hline & & gे & $\approx$ & 을 & $\tilde{N}$ & $\tilde{\kappa}$ & $\approx$ & $\approx$ & $\approx$ & مי & $\stackrel{\infty}{\sim}$ & $\approx$ & aे \\
\hline & 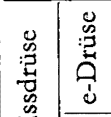 & $\stackrel{0}{n}$ & $\tilde{N}$ & 8 & $\approx$ & 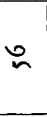 & g & \& & $\stackrel{\circ}{n}$ & $\approx$ & $F$ & n & $\approx$ \\
\hline & 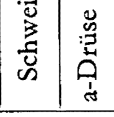 & g & $\approx$ & $\Sigma$ & q & $\stackrel{\infty}{\forall}$ & $\stackrel{m}{m}$ & $\stackrel{\infty}{m}$ & q & i & $n$ & $\tilde{\sigma}$ & 8 \\
\hline \multicolumn{2}{|c|}{ 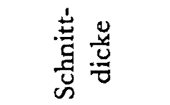 } & 함 & = & $\hat{n}$ & $\therefore$ & $=$ & $=$ & $\hat{a}$ & a & $\hat{a}$ & $\therefore$ & $\hat{a}$ & $\therefore$ \\
\hline \multicolumn{2}{|c|}{ ૧પગ્ગપગऽə૭ } & of & $\hat{2}$ & $\therefore$ & Ło & 아 & Ło & of & to & of & to & of & «o \\
\hline \multicolumn{2}{|c|}{ 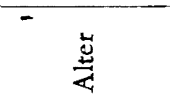 } & $m$ & $\begin{array}{l}\text { g } \\
N\end{array}$ & $\begin{array}{l}E \\
m\end{array}$ & $\begin{array}{l}E \\
m\end{array}$ & $\begin{array}{l}E \\
\infty\end{array}$ & - & $\tilde{i}$ & - & $\stackrel{-}{m}$ & 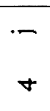 & - & $\stackrel{-}{\infty}$ \\
\hline \multicolumn{2}{|r|}{$\overline{\text { चा }}$} & r & n & m & $\forall$ & $n$ & ৩ & $r$ & $\infty$ & $a$ & 움 & $\approx$ & $\approx$ \\
\hline
\end{tabular}


Quantitative Untersuchung der Anhangsorgane der Achselhaut bei den japanischen Kindern. 427

ten Gewicht projiziert gezeichnet; dann wurden die gezeichneten Gegenstände aus allen Papierstücken sorgfältig mit der Schere herausgeschnitten und mittelst der Torsionswage genau gewogen.

Zur Berechnung des Volumens brauchte ich folgende Gleichung nach Okajima

$$
\begin{aligned}
& \mathrm{V}=\frac{\mathrm{FD} \Sigma \mathrm{W}}{\mathrm{W}_{1} \mathrm{~m}^{2}} \\
& \mathrm{~V}=\text { Volumen des Gegenstandes, } \quad \mathrm{F}=\text { Flächeninhalt des einzelnen Papierstückes, } \\
& \mathbb{W}_{1}=\text { Gewicht des einzelnen Papierstückes, } W=\text { Gewicht des Gegenstandes in einem Schnitt, } \\
& \mathrm{D}=\text { Dicke des Schnittes, } \quad \mathrm{m}=\text { Vergrösserung. }
\end{aligned}
$$

\section{Eigene Befunde.}

\section{Menge der Schweissdrüse.}

I) a-Drüse.

Das Volumen der a-Drüsen in I qcm Flächeninhalt der Achselhaut variiert nach dem Alter und individuell erheblich, wie in der Tabelle 2 und 3 angegeben. Die Drüsenmenge ist beim 8.5 jährigen Knaben $(5.03 \mathrm{I} \mathrm{cmm})$ am grössten unter den 12 untersuchten Individuen, dann folgen 8 monatiges (2.620 $\mathrm{cmm}$ ), 1.5 jähriges (1.892 cmm), 8 jähriges Mädchen $(1.623 \mathrm{cmm}), 2$ jähriger (1.494 cmm), 4 jähriger Knabe (1.010 cmm), Neugeborener von 3 Tagen $(0.894 \mathrm{cmm}), 3$ monatiges Mädchen $(0.807 \mathrm{cmm}), 3$ monatiger $(0.618 \mathrm{cmm})$,

\begin{tabular}{|c|c|c|c|c|c|c|c|}
\hline Fall & Alter & 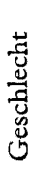 & $\begin{array}{l}\text { Wirkliches } \\
\text { Volumen } \\
\text { der a-Drüsen } \\
\text { in } \pi \mathrm{r}^{2} \text { qmm } \\
\left(\mathrm{r}=\frac{100}{50} \mathrm{~mm}\right)\end{array}$ & $\begin{array}{l}\text { Volumen der } \\
\text { a-Drüsen in } \\
\text { I qcm }\end{array}$ & $\begin{array}{l}\text { Angenom- } \\
\text { mene Aus- } \\
\text { gleichs- } \\
\text { dicke } \\
\text { (rund) }\end{array}$ & $\begin{array}{l}\text { Ausmün- } \\
\text { dungszahl } \\
\text { der } \\
\text { a-Drüsen } \\
\text { in I qcm }\end{array}$ & $\begin{array}{l}\text { Angenommenes } \\
\text { Volumen des } \\
\text { a-Drüsen- } \\
\text { individuums }\end{array}$ \\
\hline $\mathbf{I}$ & $3 t$ & 8 & O. I $12287 \mathrm{cmm}$ & $0.894 \mathrm{cmm}$ & $8.9 \mu$ & 374 & $0.00239 \mathrm{cmm}$ \\
\hline 2 & $2 \mathrm{~m}$ & 우 & 0.023838 & 0.190 & I.9 & 88 & 0.00216 \\
\hline 3 & $3 \mathrm{~m}$ & 우 & 0.101409 & 0.807 & 8.1 & 239 & 0.00338 \\
\hline 4 & $3 \mathrm{~m}$ & $\hat{\delta}$ & 0.077626 & 0.618 & 6.2 & 167 & 0.00370 \\
\hline 5 & $8 \mathrm{~m}$ & 우 & 0.329225 & 2.620 & 26.2 & 366 & 0.00716 \\
\hline 6 & I & o & 0.073266 & 0.583 & 5.8 & 183 & 0.00319 \\
\hline 7 & $1.5 \mathrm{j}$ & \& & 0.237708 & 1.892 & 18.9 & I59 & 0.01190 \\
\hline 8 & $2 j$ & $\delta$ & 0.187765 & $x .494$ & 14.9 & ISI & 0.00990 \\
\hline 9 & $3.5 \mathrm{i}$ & 우 & 0.051597 & $0.4 \mathrm{II}$ & $4 \cdot I$ & 95 & 0.00432 \\
\hline xo & $4 i$ & 令 & . 0.126889 & I.OIO & IO.I & I I I & 0.00910 \\
\hline II & $8 j$ & 우 & $0.20397^{8}$ & 1.623 & I6.2 & 95 & 0.01709 \\
\hline 12 & $8.5 \mathrm{j}$ & 今 & 0.632245 & 5.031 & 50.3 & I99 & 0.02528 \\
\hline
\end{tabular}
I jähriger Knabe $(0.583 \mathrm{cmm})$, 3.5 jähriges Mädchen $(0.4 \mathrm{II} \mathrm{cmm})$; sie ist

Tabelle 2. Menge und Ausmündungszahl der a-Drüsen in I $\mathrm{qcm}$ der Achselhaut der japanischen Kinder. 
Tabelle 3. Reihenfolge der Mengengrösse und Ausmündungszahl der a-Drüsen in I qcm der Achselhaut der japanischen Kinder.

\begin{tabular}{|c|c|c|c|c|c|}
\hline \multicolumn{2}{|c|}{$\begin{array}{l}\text { Volumen der a-Drüsen } \\
\text { in } \mathrm{I} \mathrm{qcm}\end{array}$} & \multicolumn{2}{|c|}{$\begin{array}{l}\text { Volumen des angenommenen } \\
\text { a-Drüsenindividuums }\end{array}$} & \multicolumn{2}{|c|}{$\begin{array}{l}\text { Ausmündungszahl der } \\
\text { a-Drüsen in } \mathrm{x} \text { qcm }\end{array}$} \\
\hline Fall & Volumen & Fall & Volumen & Fall & Zahl \\
\hline 12 & $5.031 \mathrm{cmm}$ & 12 & $0.02528 \mathrm{cmm}$ & I & 374 \\
\hline 5 & 2.620 & II & 0.01709 & 5 & 366 \\
\hline 7 & 1.892 & 7 & 0.01190 & 3 & 239 \\
\hline I I & 1.623 & 8 & 0.00990 & 12 & 199 \\
\hline 8 & 1.494 & IO & 0.00910 & 6 & 183 \\
\hline I0 & 1.010 & 5 & 0.00716 & 4 & 167 \\
\hline I & 0.894 & 9 & 0.00432 & 7 & 159 \\
\hline 3 & 0.807 & 4 & 0.00370 & 8 & ISI \\
\hline 4 & 0.618 & 3 & 0.00338 & Io & I I I \\
\hline 6 & 0.583 & 6 & 0.003 I9 & 9 & 95 \\
\hline 9 & $0.4 \mathrm{II}$ & I & 0.00239 & II & 95 \\
\hline 2 & 0.190 & 2 & 0.00216 & 2 & 88 \\
\hline
\end{tabular}

am geringsten beim 2 monatigen Mädchen $(0.190 \mathrm{cmm})$, so dass die relative Schwankungsbreite der a-Drüsenmenge ca. 26.5-I beträgt.

Die Zahl der Ausmündungen der a-Drüsen in $\mathrm{x}$ qcm Hautflächeninhalt bei den japanischen Kindern zeigt auch nach dem Alter und individuell erhebliche Schwankung, wie es bei den ekkrinen Schweissdrüsen (e-Drüsen) der Fall ist, so beim Neugeborenen von 3 Tagen (374) am grössten und beim 2 monatigen Mädchen (88) am geringsten, indem die relative Schwankungsbreite der Ausmündungszahl der a-Drüsen ca. 4.3-I ist (Tab. 2, 3). Obwohl die Ausmündungszahl der a-Drüsen, wie die der e-Drüsen, eine merkliche individuelle Schwankung zeigt, welche ich schon auch bei der Grösse dieser einzelnen a-Drüsen vorfinden konnte, ist sie im allgemeinen bei den älteren Kindern kleiner als bei den jüngeren, einige Ausnahmen ausgenommen.

Das Volumen des angenommenen a-Drüsenindividuums ( $\left.\frac{\text { Drüsenmenge in } 1 \mathrm{qcm}}{\text { Ausmündungszahl in } 1 \mathrm{qcm}}\right)$ stimmt mit dem durchschnittlichen Volumen der einzelnen a-Drüsen desselben Materials, am welch letzteren ich früher die Grösse je einer a-Drüse genau gemessen und publiziert habe, im grossen und ganzen überein (Tab. 4). Dieses angenommene Volumen schwankt auch nach dem Alter und individuell bedeutend, so ist es beim 8.5 jährigen Knaben $(0.02528 \mathrm{cmm})$ am grössten, dann kommen 8 jähriges (0.01709 cmm), I.s jähriges Mädchen (0.01190 cmm), 2 jähriger $(0.00990 \mathrm{cmm}), 4$ jähriges Knabe $(0.009$ Io $\mathrm{cmm}), 8$ monatiges $(0.00716 \mathrm{cmm}), 3.5$ jähriges Mädchen $(0.00432 \mathrm{cmm}), 3$ monatiger Knabe $(0.00370 \mathrm{cmm}), 3$ monatiges Mädchen $(0.00338 \mathrm{cmm})$, I jähriger Knabe $(0.00319 \mathrm{cmm})$, Neugeborener von 3 Tagen $(0.00239 \mathrm{cmm})$, und beim 2 monatigen Mädchen $(0.00216 \mathrm{cmm})$ ist es am kleinsten; also beträgt die relative Schwankungsbreite ca. I I.7-I (Tab. 2-4). 
Tabelle 4 .

\begin{tabular}{|c|c|c|}
\hline Fall & $\begin{array}{c}\text { Wirkliches, durchschnittliches } \\
\text { Volumen des a-Drüsenindividuums }\end{array}$ & $\begin{array}{c}\text { Angenommenes Volumen } \\
\text { des a-Drüsenindividuums }\end{array}$ \\
\hline I & $0.00203 \mathrm{cmm}$ & $0.00239 \mathrm{cmm}$ \\
2 & 0.00233 & 0.00216 \\
3 & 0.00299 & 0.00338 \\
4 & 0.00363 & 0.00370 \\
5 & 0.00596 & 0.00716 \\
6 & 0.00308 & 0.00319 \\
7 & 0.01234 & 0.01190 \\
8 & 0.01030 & 0.00990 \\
9 & 0.00414 & 0.00432 \\
I0 & 0.00964 & 0.00910 \\
I I & 0.01525 & 0.01709 \\
I2 & 0.01896 & 0.02528 \\
\hline
\end{tabular}

Die genaueren gegenseitigen Verhältnisse zwischen der Menge des angenommenen a-Drüsenindividuums, a-Drüsenmenge und Zahl der aDrüsenausmündung in einem bestimmten Hautflächeninhalt verhalten sich wie in der Tabelle 3 angegeben. Daraus kann man wohl schliessen, dass die Ausmündungszahl der a-Drüsen in einem bestimmten Hautflächeninhalt bei jüngeren Individuen im grossen und ganzen grösser als bei älteren zu sein pflegt, während das Volumen des angenommenen a-Drüsenindividuums und die a-Drüsenmenge in einem bestimmten Hautflächeninhalt im allgemeinen fast umgekehrt sich verhalten.

Das Volumen der a-Drüse, welche mit einer Haargruppe im Zusammenhang steht, wurde bei den bei jedem Fall absichtlos gewählten to Haargruppen untersucht; näheres darüber ist in den Tabellen 5-16 angegeben.

Das Volumen der a-Drüse, die mit einer einhaarigen Haargruppe im Zusammenhang steht, ist beim Fall I $2(0.036808 \mathrm{cmm})$ am grössten und beim Fall I $(0.001402 \mathrm{cmm})$ am kleinsten. Die a-Drüsen, die mit einer zweihaarigen Haargruppe im Zusammenhang stehen, haben beim Fall I 2 die grösste Menge $(0.039602 \mathrm{cmm})$ und beim Fall 9 die kleinste $(0.001139 \mathrm{cmm})$. Das Volumen der Drüsen, welche mit einer dreihaarigen Haargruppe im Zuşammenhang stehen, sind beim Fall in $(0.035187 \mathrm{cmm})$ am grössten und beim Fall $9(0.000657 \mathrm{cmm})$ am kleinsten. Die a-Drüsen, die zur vierhaarigen Haargruppe gehören, haben beim Fall 7 das grösste Volumen $(0.21669 \mathrm{cmm})$ und beim Fall 2 das kleinste $(0.002443 \mathrm{cmm})$. Das Volumen der zu einer fünfhaarigen Haargruppe gehörigen Drüsen ist beim Fall 7 am grössten $(0.023783 \mathrm{cmm})$ und beim Fall 6 am kleinsten $(0.002596 \mathrm{cmm})$.

\section{2) e-Drüse.}

Das Volumen der e-Drüsen in I qcm der Achselhaut variiert nach dem Alter und individuell, wie Tabelle $\mathrm{I} 7$ und $\mathrm{I} 8$ es zeigen. Bei den $\mathrm{I} 2$ untersuchten 
Tabelle 5-I6. Volumen der zu einer Haargruppe gehörigen a-Drüsen bei den einzelnen Fällen.

Tabelle 5. Fall I.

\begin{tabular}{|c|c|c|}
\hline $\begin{array}{c}\text { Haar- } \\
\text { gruppe }\end{array}$ & $\begin{array}{c}\text { Haarzahl } \\
\text { in einer } \\
\text { Gruppe }\end{array}$ & $\begin{array}{c}\text { Volumen } \\
\text { der Drüsen }\end{array}$ \\
\hline a & 2 & $0.005532 \mathrm{cmm}$ \\
b & 2 & 0.001534 \\
c & I & $0.00185 \mathrm{I}$ \\
d & 3 & $0.00322 \mathrm{I}$ \\
e & 2 & 0.003933 \\
f & 3 & $0.00712 \mathrm{I}$ \\
$\mathrm{g}$ & 2 & 0.001490 \\
$\mathrm{~h}$ & $\mathrm{I}$ & 0.001402 \\
$\mathrm{i}$ & 3 & 0.003265 \\
$\mathrm{j}$ & 2 & 0.002344 \\
\hline
\end{tabular}

Tabelle 7. Fall 3.

\begin{tabular}{|c|c|c|}
\hline $\begin{array}{c}\text { Haar- } \\
\text { gruppe }\end{array}$ & $\begin{array}{c}\text { Haarzahl } \\
\text { in einer } \\
\text { Gruppe }\end{array}$ & $\begin{array}{c}\text { Volumen } \\
\text { der Drüsen }\end{array}$ \\
\hline $\mathrm{a}$ & 2 & $0.002246 \mathrm{cmm}$ \\
$\mathrm{b}$ & 3 & 0.003637 \\
$\mathrm{c}$ & 3 & 0.007329 \\
$\mathrm{~d}$ & 2 & 0.002520 \\
$\mathrm{e}$ & 2 & 0.003703 \\
$\mathrm{f}$ & 2 & 0.004294 \\
$\mathrm{~g}$ & 3 & 0.001358 \\
$\mathrm{~h}$ & 3 & 0.002399 \\
$\mathrm{i}$ & 2 & 0.002202 \\
$\mathrm{j}$ & 3 & 0.002629 \\
\hline
\end{tabular}

Tabelle 9. Fall s.

\begin{tabular}{|c|c|c|}
\hline $\begin{array}{c}\text { Haar- } \\
\text { gruppe }\end{array}$ & $\begin{array}{c}\text { Haarzahl } \\
\text { in einer } \\
\text { Gruppe }\end{array}$ & $\begin{array}{c}\text { Volumen } \\
\text { der Drüsen }\end{array}$ \\
\hline $\mathrm{a}$ & 2 & $0.011042 \mathrm{cmm}$ \\
$\mathrm{b}$ & 3 & 0.005247 \\
$\mathrm{c}$ & 3 & 0.013945 \\
$\mathrm{~d}$ & 3 & 0.015337 \\
$\mathrm{e}$ & 2 & 0.005850 \\
$\mathrm{f}$ & 3 & 0.013967 \\
$\mathrm{~g}$ & 4 & 0.005466 \\
$\mathrm{~h}$ & 4 & 0.009542 \\
$\mathrm{i}$ & 3 & 0.007405 \\
$\mathrm{j}$ & 3 & 0.012719 \\
\hline
\end{tabular}

Tabelle 6. Fall 2.

\begin{tabular}{|c|c|c|}
\hline $\begin{array}{c}\text { Haar- } \\
\text { gruppe }\end{array}$ & $\begin{array}{c}\text { Haarzahl } \\
\text { in einer } \\
\text { Gruppe }\end{array}$ & $\begin{array}{c}\text { Volumen } \\
\text { der Drüsen }\end{array}$ \\
\hline $\mathrm{a}$ & 3 & $0.001238 \mathrm{cmm}$ \\
$\mathrm{b}$ & 3 & 0.001194 \\
$\mathrm{c}$ & 2 & 0.003013 \\
$\mathrm{~d}$ & 3 & 0.002290. \\
$\mathrm{e}$ & 4 & 0.002443 \\
$\mathrm{f}$ & 3 & 0.002322 \\
$\mathrm{~g}$ & 2 & 0.002290 \\
$\mathrm{~h}$ & 3 & 0.002136 \\
$\mathrm{i}$ & 2 & 0.003045 \\
$\mathrm{j}$ & 3 & 0.002520 \\
\hline
\end{tabular}

Tabelle 8. Fall 4.

\begin{tabular}{|c|c|c|}
\hline $\begin{array}{c}\text { Haar- } \\
\text { gruppe }\end{array}$ & $\begin{array}{c}\text { Haarzahl } \\
\text { in einer } \\
\text { Gruppe }\end{array}$ & $\begin{array}{c}\text { Volumen } \\
\text { der Drüsen }\end{array}$ \\
\hline a & 2 & $0.002103 \mathrm{cmm}$ \\
b & 2 & 0.006189 \\
c & 3 & 0.000898 \\
d & 3 & 0.002246 \\
e & 3 & $0.00494 \mathrm{I}$ \\
f & 2 & 0.001150 \\
g & 2 & 0.002728 \\
h & 3 & 0.007668 \\
i & 2 & 0.004437 \\
i & 3 & 0.003122 \\
\hline
\end{tabular}

Tabelle ıo. Fall 6.

\begin{tabular}{|c|c|c|}
\hline $\begin{array}{c}\text { Haar- } \\
\text { gruppe }\end{array}$ & $\begin{array}{c}\text { Haarzahl } \\
\text { in einer } \\
\text { Gruppe }\end{array}$ & $\begin{array}{c}\text { Volumen } \\
\text { der Drüsen }\end{array}$ \\
\hline a & 2 & 0.00426 I cmm \\
b & 3 & 0.0035 16 \\
c & 4 & 0.00483 I \\
d & 4 & 0.004174 \\
e & 2 & 0.003703 \\
f & 5 & 0.002596 \\
g & 3 & 0.003604 \\
h & 3 & 0.002684 \\
i & 4 & 0.006847 \\
j & 5 & 0.003308 \\
\hline
\end{tabular}


Quantitative Untersuchung der Anhangsorgane der Achselhaut bei den japanischen Kindern. 43 I

Tabelle i $\mathrm{r}$. Fall 7 .

\begin{tabular}{|c|c|c|}
\hline $\begin{array}{c}\text { Haar- } \\
\text { gruppe }\end{array}$ & $\begin{array}{c}\text { Haarzahl } \\
\text { in einer } \\
\text { Gruppe }\end{array}$ & $\begin{array}{c}\text { Volumen } \\
\text { der Drüsen }\end{array}$ \\
\hline $\mathrm{a}$ & 5 & $0.023783 \mathrm{cmm}$ \\
$\mathrm{b}$ & 3 & 0.014165 \\
$\mathrm{c}$ & 2 & 0.010955 \\
$\mathrm{~d}$ & 3 & 0.012883 \\
$\mathrm{e}$ & 2 & 0.007526 \\
$\mathrm{f}$ & 2 & 0.015852 \\
$\mathrm{~g}$ & 2 & 0.016673 \\
$\mathrm{~h}$ & 4 & 0.021669 \\
$\mathrm{i}$ & $\mathrm{I}$ & 0.006945 \\
$\mathbf{j}$ & 3 & 0.021570 \\
\hline
\end{tabular}

Tabelle 13. Fall 9.

\begin{tabular}{|c|c|c|}
\hline $\begin{array}{c}\text { Haar- } \\
\text { gruppe }\end{array}$ & $\begin{array}{c}\text { Haarzahl } \\
\text { in einer } \\
\text { Gruppe }\end{array}$ & $\begin{array}{c}\text { Volumen } \\
\text { der Drüsen }\end{array}$ \\
\hline a & 3 & $0.005740 \mathrm{cmm}$ \\
b & 2 & 0.001139 \\
c & 3 & 0.005762 \\
d & 2 & 0.004393 \\
e & 3 & 0.010254 \\
f & 3 & 0.000657 \\
g & 2 & 0.002629 \\
h & 3 & 0.005291 \\
i & 3 & 0.006967 \\
j & 3 & 0.002980 \\
\hline
\end{tabular}

Tabelle is. Fall in.

\begin{tabular}{|c|c|c|}
\hline $\begin{array}{c}\text { Haar- } \\
\text { gruppe }\end{array}$ & $\begin{array}{c}\text { Haarzahl } \\
\text { in einer } \\
\text { Gruppe }\end{array}$ & $\begin{array}{c}\text { Volumen } \\
\text { der Drüsen }\end{array}$ \\
\hline $\mathrm{a}$ & 2 & $0.018032 \mathrm{cmm}$ \\
$\mathrm{b}$ & 2 & 0.007657 \\
$\mathrm{c}$ & $\mathrm{I}$ & 0.015446 \\
$\mathrm{~d}$ & 2 & 0.015337 \\
$\mathrm{e}$ & 4 & 0.006310 \\
$\mathrm{f}$ & 2 & 0.008578 \\
$\mathrm{~g}$ & 2 & $0.02353 \mathrm{I}$ \\
$\mathrm{h}$ & 2 & 0.022435 \\
$\mathrm{i}$ & 3 & $0.035 \mathrm{I} 87$ \\
$\mathrm{j}$ & 3 & 0.016575 \\
\hline
\end{tabular}

Tabelle $12 . \quad$ Fall 8.

\begin{tabular}{|c|c|c|}
\hline $\begin{array}{c}\text { Haar- } \\
\text { gruppe }\end{array}$ & $\begin{array}{c}\text { Haarzahl } \\
\text { in einer } \\
\text { Gruppe }\end{array}$ & $\begin{array}{c}\text { Volumen } \\
\text { der Drüsen }\end{array}$ \\
\hline a & 2 & $0.007877 \mathrm{cmm}$ \\
$\mathrm{b}$ & 2 & 0.016334 \\
$\mathrm{c}$ & 3 & $0.01343 \mathrm{I}$ \\
$\mathrm{d}$ & 3 & 0.016695 \\
$\mathrm{e}$ & 3 & 0.008884 \\
$\mathrm{f}$ & 2 & 0.016180 \\
$\mathrm{~g}$ & 2 & 0.009333 \\
$\mathrm{~h}$ & 5 & 0.013617 \\
i & 3 & 0.022928 \\
$\mathrm{j}$ & 2 & 0.016838 \\
\hline
\end{tabular}

Tabelle I4. Fall io.

\begin{tabular}{|c|c|c|}
\hline $\begin{array}{c}\text { Haar- } \\
\text { gruppe }\end{array}$ & $\begin{array}{c}\text { Haarzahl } \\
\text { in einer } \\
\text { Gruppe }\end{array}$ & $\begin{array}{c}\text { Volumen } \\
\text { der Drüsen }\end{array}$ \\
\hline $\mathrm{a}$ & 2 & $0.001610 \mathrm{cmm}$ \\
$\mathrm{b}$ & 2 & 0.012970 \\
$\mathrm{c}$ & 3 & 0.009585 \\
$\mathrm{~d}$ & 2 & 0.008096 \\
$\mathrm{e}$ & 2 & 0.008585 \\
$\mathrm{f}$ & $\mathrm{I}$ & 0.007920 \\
$\mathrm{~g}$ & 3 & $0.01493 \mathrm{I}$ \\
$\mathrm{h}$ & 2 & 0.010955 \\
$\mathrm{i}$ & 3 & 0.012927 \\
$\mathrm{j}$ & 2 & $0.00827 \mathrm{I}$ \\
\hline
\end{tabular}

Tabelle I6. Fall 12.

\begin{tabular}{|c|c|c|}
\hline $\begin{array}{c}\text { Haar- } \\
\text { gruppe }\end{array}$ & $\begin{array}{c}\text { Haarzahl } \\
\text { in einer } \\
\text { Gruppe }\end{array}$ & $\begin{array}{c}\text { Volumen } \\
\text { der Drüsen }\end{array}$ \\
\hline a & 2 & $0.017845 \mathrm{cmm}$ \\
b & 2 & 0.006737 \\
c & 3 & 0.018853 \\
d & I & 0.036808 \\
e & 2 & 0.039602 \\
f & 2 & 0.01148 I \\
g & 2 & 0.010988 \\
h & 2 & 0.011283 \\
i & 2 & 0.020113 \\
j & 3 & 0.015917 \\
\hline
\end{tabular}

Individuen ist die Drüsenmenge beim 8 jährigen Mädchen $(6.729 \mathrm{cmm}) \mathrm{am}$ grössten, dann folgen 8.5 jähriger (6.25 I cmm), 2 jähriger Knabe ( $4.2 \mathrm{I} 2 \mathrm{cmm}$ ), 3 monatiges (4.16I cmm), 8 monatiges $(4.087 \mathrm{cmm}$ ), 3.5 jähriges Mädchen $(3.987 \mathrm{cmm}), 3$ monatiger $(3.886 \mathrm{cmm}), 4$ jähriger $(3.426 \mathrm{cmm})$, I jähriger 
Tabelle I7. Menge und Ausmündungszahl der e-Drüsen in I qcm der Achselhaut der japanischen Kinder.

\begin{tabular}{|c|c|c|c|c|c|c|c|}
\hline Fall & Alter & 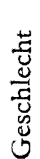 & $\begin{array}{l}\text { Wirkliches } \\
\text { Volumen der } \\
\text { e-Drüsen } \\
\text { in } \pi \mathrm{r}^{2} \mathrm{qmm} \\
(\mathrm{r}=\stackrel{100}{=} \mathrm{mm})\end{array}$ & $\begin{array}{l}\text { Volumen der } \\
\text { e-Drüsen in } \\
\text { I qcm }\end{array}$ & $\begin{array}{l}\text { Angenom- } \\
\text { mene Aus- } \\
\text { gleichsdicke } \\
\text { (rund) }\end{array}$ & $\begin{array}{c}\text { Ausmün- } \\
\text { dungszahl } \\
\text { der e-Drüsen } \\
\text { in I qcm }\end{array}$ & $\begin{array}{l}\text { Angenommenes } \\
\text { Volumen des } \\
\text { e-Drüsen- } \\
\text { individuums }\end{array}$ \\
\hline I & $\mathbf{t}$ & f & $0.32964 \mathrm{rcmm}$ & $2.623 \mathrm{cmm}$ & $26.2 \mu$ & 1339 & $0.00196 \mathrm{cmm}$ \\
\hline 2 & $2 \mathrm{~m}$ & 우 10 & 0.198085 & I. $57^{6}$ & 15.8 & 756 & 0.00208 \\
\hline 3 & $\mathrm{~m}$ & 우 & 0.522927 & $4.16 \mathrm{I}$ & 41.6 & 1369 & 0.00304 \\
\hline 4 & $\mathrm{~m}$ & $\delta$ & 0.488299 & 3.886 & 38.9 & 923 & $0.0042 \mathrm{I}$ \\
\hline 5 & $\mathrm{~m}$ & 우 & 0.513649 & 4.087 & 40.9 & 756 & $0.0054 \mathrm{I}$ \\
\hline 6 & I j & 今 & $0.4 \mathrm{I} 500 \mathrm{I}$ & 3.302 & 33.0 & I I 54 & 0.00286 \\
\hline 7 & 1.5 & 우 & 0.308706 & 2.457 & 24.6 & 414 & 0.00593 \\
\hline 8 & 2 & 今 & $0.52928 \mathrm{I}$ & 4.212 & 42.1 & 525 & 0.00802 \\
\hline 9 & 3.5 & q & 0.500974 & $3.9^{87}$ & 39.9 & 517 & $0.0077^{I}$ \\
\hline Io & & 今 & 0.430567 & 3.426 & 34.3 & 587 & 0.00884 \\
\hline I I & & 우 & 0.845623 & 6.729 & 67.3 & 597 & 0.01127 \\
\hline I 2 & $8.5 \mathrm{j}$ & 今 & $0.7^{8548} \mathrm{I}$ & $6.25 \mathrm{I}$ & 62.5 & 589 & $0.0106 \mathrm{I}$ \\
\hline
\end{tabular}

Tabelle 18 . Reihenfolge der Mengengrösse und Ausmündungszahl der e-Drüsen in I qcm der Achselhaut der japanischen Kinder.

\begin{tabular}{|c|c|c|c|c|c|}
\hline \multicolumn{2}{|c|}{$\begin{array}{l}\text { Volumen der e-Drüsen } \\
\text { in } \mathrm{I} \mathrm{qcm}\end{array}$} & \multicolumn{2}{|c|}{$\begin{array}{l}\text { Volumen des angenommenen } \\
\text { Drüsenindividuums }\end{array}$} & \multicolumn{2}{|c|}{$\begin{array}{l}\text { Ausmündungszahl der } \\
\text { e-Drüsen in } \mathrm{I} \mathrm{gcm}\end{array}$} \\
\hline Fall & Volumen & Fall & Volumen & Fall & Zahl \\
\hline I I & $6.729 \mathrm{cmm}$ & I I & 0.01 I $27 \mathrm{cmm}$ & 3 & 1369 \\
\hline I 2 & $6.25 \mathrm{I}$ & I 2 & $0.0106 \mathrm{I}$ & I & 1339 \\
\hline 8 & 4.2 I 2 & 8 & 0.00802 & 6 & I I 54 \\
\hline 3 & $4.16 \mathrm{I}$ & 9 & 0.00771 & 4 & 923 \\
\hline 5 & 4.087 & 7 & 0.00593 & 5 & 756 \\
\hline 9 & 3.987 & 10 & 0.00584 & 2 & 756 \\
\hline 4 & 3.886 & 5 & 0.00541 & XI & 597 \\
\hline 10 & 3.426 & 4 & $0.0042 \mathrm{I}$ & I 2 & 589 \\
\hline 6 & 3.302 & 3 & 0.00304 & Io & 587 \\
\hline I & 2.623 & 6 & 0.00286 & 8 & 525 \\
\hline 7 & 2.457 & 2 & 0.00208 & 9 & 517 \\
\hline 2 & 1.576 & I & 0.00196 & 7 & $4 \mathrm{I} 4$ \\
\hline
\end{tabular}

Knabe $(3.302 \mathrm{cmm})$, Neugeborener von 3 Tagen $(2.623 \mathrm{cmm})$, I.5 jähriges Mädchen $(2.457 \mathrm{cmm})$; sie ist beim 2 monatigen Mädchen $(1.576 \mathrm{cmm}) \mathrm{am}$ kleinsten, so dass die relative Schwankungsbreite der Menge ca. 4.3-I beträgt. Also ist die relative Schwankungsbreite der e-Drüsenmenge in $\mathrm{r} \mathrm{qcm}$ weit kleiner als die der a-Drüsenmenge (26.5-I).

Vergleicht man die Befunde von Taniguchi (Neugeborene) und Mine (9 monatiger) mit meinen Befunden bei den Kindern im gleichen Alter, so findet man, dass das Volumen der e-Drüsen in I qcm bei meinem Fall I (Neugeborene) $(2.623 \mathrm{cmm})$ dem am Bauch der Neugeborenen von Taniguchi 
$(2.704 \mathrm{cmm})$ entspricht, welches unter den is untersuchten Körperteilen am geringsten ist, und dass das Volumen beim Fall 5 ( 8 monatiger $)(4.086 \mathrm{cmm})$ mit dem an der Brust des 9 monatigen von Mine $(4.006 \mathrm{cmm})$ fast übereinstimmt, welches unter den is untersuchten Körperteilen das kleinste ist.

Die Zahl der Ausmündungen der e-Drüsen schwankt auch nach dem Alter und individuell erheblich, so ist sie in I qcm am grössten beim 3 monatigen Mädchen ( 1369 ) und am kleinsten beim I.s jährigen Mädchen (414); also ist die relative Schwankungsbreite ca. 3.3-I (Tab. I7, 18). Die relative Schwankungsbreite der Ausmündungszahl ist bei den e-Drüsen etwas kleiner als bei den a-Drüsen (4.3-I).

Wenn man vorläufig annimmt, dass eine e-Drüse nur eine Ausmündung besitzt, so lässt sich das Volumen der einzelnen e-Drüse folgendermassen leicht berechnen :

$$
\frac{\text { Menge der e-Drüsen in I qcm }}{\text { Zahl der Ausmündungen in I qcm }}
$$

Das Volumen dieses angenommenen e-Drüsenindividuums ist auch nach dem Alter und individuell verschieden gross, so beim 8 jährigen Mädchen am grössten $(0.01 \mathrm{I} 27 \mathrm{cmm})$ und beim Neugeborenen von 3 Tagen am kleinsten $(0.00196 \mathrm{cmm}$ ) (Tab. 17, 18), also beträgt die relative Schwankungsbreite, welche viel kleiner als die bei den a-Drüsen (I I.7-I) ist, ca. 5.7-I.

Der Vergleich der relativen Schwankungsbreite der a- und e-Drüsenmenge in I qcm, Ausmündungszahl der a- und e-Drüsen in I $\mathrm{qcm}$ sowie des angenommenen Volumens des a- und e-Drüsenindividuums erhellt, dass die individuelle Schwankung des Entwicklungsgrades bei den a-Drüsen auffallend grösser als bei den e-Drüsen ist.

Die gegenseitigen Verhältnisse der Menge des e-Drüsenindividuums, der der e-Drüsen und der Zahl der e-Drüsenausmündung in einem bestimmten Hautflächeninhalt der Achselhaut sind in der Tabelle I 8 angegeben. Daraus geht hervor, dass die Menge der e-Drüsen in I $\mathrm{qcm}$ und das Volumen des angenommenen e-Drüsenindividuums an der Achselhaut bei den älteren Kindern im grossen und ganzen grösser als bei den jüngeren sind und es bei der e-Drüsenausmündungszahl demgegenüber meistens umgekehrt der Fall ist, obwohl dabei eine ziemlich grosse individuelle Schwankung vorkommt.

Bezüglich der Proportion zwischen der in I qcm Flächeninhalt an der Achselhaut befindlichen a- und e-Drüsenmenge ist das Volumen der e-Drüsen bei allen untersuchten Fällen grösser als das der a-Drüsen (Tab. I9). Die Proportion zwischen beiden Drüsenmengen (a-Dr./e-Dr.) ist auch individuell sehr variabel, indem sie beim Fall 9 (I/9.7) am kleinsten, beim Fall 
I 2 (I/I.2) am grössten ist, und keinen Zusammenhang mit dem Kindesalter zeigt.

Tabelle 19.

\begin{tabular}{|c|c|c|c|}
\hline \multirow{2}{*}{ Fall } & \multicolumn{2}{|c|}{ Volumen in $\mathrm{I} \mathrm{qcm}$} & \multirow{2}{*}{$\frac{a-D r}{e-D r}$} \\
\hline & a-Drüsen & e-Drüsen & \\
\hline I & $0.89355 \mathrm{cmm}$ & $2.62319 \mathrm{cmm}$ & I / 2.9 \\
\hline 2 & I.I 8970 & 1.57631 & I / 8.3 \\
\hline 3 & 0.80699 & 4.16131 & I $/ 5.2$ \\
\hline 4 & 0.61773 & 3.88575 & I / 6.3 \\
\hline 5 & 2.61988 & 4.08747 & $1 / 1.6$ \\
\hline 6 & 0.58303 & 3.30347 & I $/ 5.7$ \\
\hline 7 & 1.89162 & 2.45660 & $1 / 1.3$ \\
\hline 8 & I. 494 I 8 & $4.2 \mathrm{I} \times 88$ & I / 2.8 \\
\hline 9 & 0.41060 & 3.98662 & I $/ 9.7$ \\
\hline Io & I.00975 & 3.42634 & I $/ 3.4$ \\
\hline I I & 1.62320 & 6.72924 & $I / 4 . I$ \\
\hline I 2 & 5.03123 & 6.25066 & $\mathrm{I} / \mathrm{I} .2$ \\
\hline
\end{tabular}

Tabelle 20.

\begin{tabular}{|c|c|c|c|}
\hline \multirow{2}{*}{ Fall } & \multicolumn{2}{|c|}{$\begin{array}{l}\text { Volumen des angenommenen } \\
\text { Drüsenindividuums }\end{array}$} & \multirow{2}{*}{$\frac{a-D r}{e-D r .}$} \\
\hline & a-Drüsen & e-Drüsen & \\
\hline I & $0.00239 \mathrm{cmm}$ & $0.0019^{6} \mathrm{cmm}$ & I $/ 0.82$ \\
\hline 2 & 0.00216 & 0.00208 & I / 0.96 \\
\hline 3 & 0.00338 & 0.00304 & I / 0.90 \\
\hline 4 & 0.003 & 0.0042 & I $/ \mathrm{I} . \mathrm{I} 4$ \\
\hline 5 & 0.00716 & 0.005 & $x / 0.76$ \\
\hline 6 & 0.003 & 0.00286 & I / 0.90 \\
\hline 7 & 0.01190 & 0.00 & $1 / 0.50$ \\
\hline 8 & 0.00990 & 0.00802 & $\mathrm{I} / 0.8 \mathrm{I}$ \\
\hline 9 & 0.00432 & 0.00771 & I $/ 1.7^{8}$ \\
\hline 10 & 0.00910 & 0.00584 & I / 0.64 \\
\hline II & 0.01709 & $0.01 \mathrm{I}$ & $1 / 0.66$ \\
\hline I 2 & 0.02528 & $0.0106 \mathrm{I}$ & I $/ 0.42$ \\
\hline
\end{tabular}

Die Tabelle 20 zeigt die Proportion zwischen dem angenommenen Volúmen $\left(\frac{\text { Drüsenmenge in I } \mathrm{qcm}}{\text { Ausmündungszahl in I } \mathrm{qcm}}\right)$ des a- und e-Drüsenindividuums. Das angenommene Volumen des a-Drüsenindividuums ist ebenfalls etwas grösser als das des e-Drüsenindividuums, abgesehen von zwei Ausnahmefällen (Fall 4 und 9). Die Proportion des Volumens des angenommenen Drüsenindividuums zwischen beiden Drüsenarten (a-Dr./e-Dr.) ist beim Fall 9 am kleinsten $(\mathrm{I} / \mathrm{r} .78)$ und beim Fall $\mathrm{I} 2 \mathrm{am}$ grössten $(\mathrm{I} / \mathrm{0.42})$.

Vergleicht man die Ausmündungszahl der a- und e-Drüsen in I $\mathrm{q} c \mathrm{~m}$, so findet man, dass die Ausmündungszahl der e-Drüsen ausnahmslos grösser als die der a-Drüsen ist (Tab. 21). Die Proportion der Ausmündungszahl zwischen beiden Drüsenarten (a-Dr./e-Dr.) ist beim Fall 2 am kleinsten $(\mathrm{I} / 8.6)$ und beim Fall 5 am grössten (I/2.I).

Das Volumen des angenommenen c-Drüsenindividuums bei meinem Fall Tabelle 2I.

\begin{tabular}{|c|c|c|c|}
\hline \multirow{2}{*}{ Fall } & \multicolumn{2}{|c|}{ Ausmündungszahl in $\mathrm{r} \mathrm{qcm}$} & \multirow{2}{*}{$\frac{a-D r}{\text { e-Dr. }}$} \\
\hline & a-Drüsen & e-Drüsen & \\
\hline I & 374 & 1339 & $x / 3.6$ \\
\hline 2 & 88 & 756 & I $/ 8.6$ \\
\hline 3 & 239 & I369 & I $/ 5.7$ \\
\hline 4 & 167 & 923 & I $/ 5.4$ \\
\hline 5 & 366 & 756 & $I / 2 . I$ \\
\hline 6 & 183 & I I 54 & I / 6.3 \\
\hline 7 & 159 & 414 & $1 / 2.6$ \\
\hline 8 & IS I & 525 & I / 3.5 \\
\hline 9 & 95 & 517 & I $/ 5.4$ \\
\hline Io & I I I & 587 & I $/ 5.3$ \\
\hline I I & 95 & 597 & $1 / 6.3$ \\
\hline I 2 & 199 & 589 & $1 / 3.0$ \\
\hline
\end{tabular}
I (Neugeborener) $(0.00196 \mathrm{cmm})$ ist sehr klein und entspricht ca. I/I.3 des kleinsten Volumens (Bauch, 0.002589 
Quantitative Untersuchung der Anhangsorgane der Achselhaut bei den japanischen Kindern. 435

$\mathrm{cmm}$ ) bei der Neugeborenen von Taniguchi. Das Volumen bei meinem Fall s (8 monatige) $(0.0054 \mathrm{I} \mathrm{cmm})$ ist auch sehr klein und liegt in der Nähe des kleinsten Volumens beim 9 monatigen Knaben von Mine (Lateralseite des Oberschenkels, $0.005084 \mathrm{cmm}$ ).

Ordnet man jeden Fall nach der Reihenfolge der Grösse der Menge oder Ausmündungszahl der a- sowie e-Drüsen an, so bekommt man die Tabelle 22. Bezüglich der Reihenfolge der Grösse des angenommenen Volumens des a- und e-Drüsenindividuums findet man einen Parallelismus zwischen beiden Drüsenarten, nämlich bei einem Fall, bei welchem das angenommene Volumen des a-Drüsenindividuums grösser ist, ist auch das des e-Drüsenindividuums grösser, während man bei der Reihenfolge des Volumens und

Tabelle 22. Vergleich der Reihenfolge der Mengengrösse und Ausmündungszahl zwischen den a- und e-Drüsen an der Achselhaut der japanischen Kinder.

\begin{tabular}{|c|c|c|c|c|c|c|c|c|c|c|c|}
\hline \multicolumn{4}{|c|}{ Volumen in $\mathrm{x} \mathrm{qcm}$} & \multicolumn{4}{|c|}{$\begin{array}{l}\text { Volumen des angenommenen } \\
\text { Drüsenindividuums }\end{array}$} & \multicolumn{4}{|c|}{$\begin{array}{l}\text { Ausmündungszahl } \\
\text { in I } \mathrm{qcm}\end{array}$} \\
\hline \multicolumn{2}{|c|}{ a-Drüsen } & \multicolumn{2}{|c|}{ e-Drüsen } & \multicolumn{2}{|c|}{ a-Drüsen } & \multicolumn{2}{|c|}{ e-Drüsen } & \multicolumn{2}{|c|}{ a-Drüsen } & \multicolumn{2}{|c|}{ e-Drüsen } \\
\hline Fall & Volumen & Fall & Volumen & Fall & Volumen & Fall & Volumen & Fall & Zahl & Fall & Zahl \\
\hline I 2 & $5.031 \mathrm{cmm}$ & I I & $6.729 \mathrm{cmm}$ & I 2 & $0.025 \mathrm{cmm}$ & II & $0.01 \mathrm{I} \mathrm{cmm}$ & I & 374 & 3 & 1369 \\
\hline 5 & 2.620 & I 2 & $6.25 \mathrm{I}$ & I I & 0.017 & 12 & 0.011 & 5 & 366 & I & I 339 \\
\hline 7 & 1.892 & 8 & 4.212 & 7 & 0.012 & 8 & 0.008 & 3 & 239 & 6 & II 54 \\
\hline II & 1.623 & 3 & $4.16 \mathrm{I}$ & 8 & 0.010 & 9 & 0.008 & 12 & I99 & 4 & 923 \\
\hline 8 & I. 494 & 5 & 4.087 & IO & 0.009 & 7 & 0.006 & 6 & 183 & 5 & 756 \\
\hline 10 & 1.010 & 9 & 3.987 & 5 & 0.007 & Io & 0.006 & 4 & 167 & 2 & 756 \\
\hline I & 0.894 & 4 & 3.886 & 9 & 0.004 & 5 & 0.005 & 7 & 159 & I I & 597 \\
\hline 3 & 0.807 & 10 & 3.426 & 4 & 0.004 & 4 & 0.004 & 8 & 151 & I 2 & 589 \\
\hline 4 & 0.618 & 6 & 3.302 & 3 & 0.003 & 3 & 0.003 & 10 & I I I & IO & 587 \\
\hline 6 & 0.583 & I & 2.623 & 6 & 0.003 & 6 & 0.003 & 9 & 95 & 8 & 525 \\
\hline 9 & 0.4 I I & 7 & 2.457 & I & 0.002 & 2 & 0.002 & II & 95 & 9 & 517 \\
\hline 2 & 0.190 & 2 & 1.576 & 2 & 0.002 & I & 0.002 & 2 & 88 & 7 & 414 \\
\hline
\end{tabular}

der Ausmündungszahl in I qcm den Parallelismus zwischen a- und e-Drüsen kaum vorfinden kann.

Im Folgenden werde ich die Ausmündungszahl der e-Drüsen in I qcm der Achselhaut mit der der anderen Körperteile bei Kindern im gleichen Alter (Koyama) vergleichen (Tab. 23). Nach Koyama befindet sich die grösste Zahl der Ausmündung der e-Drüsen in I qcm bei einer Neugeborenen an der Beugeseite des Unterschenkels (232I) und die kleinste an der Brust (IоI8). Die Ausmündungszahl an der Achselhaut bei meinem gleichaltrigen Material (Fall I) (1 369) ist fast gleich gross wie diejenige am Gesässe (I 370 ) der Neugeborenen von K o y a ma und liegt etwa an der Grenze des kleineren Zwei- und Dreisiebentels der Schwankungsbreite bei der letzteren. Die 
Ausmündungszahl beim 2 monatigen Kind von $\mathrm{K}$ oy a ma befindet sich zwischen I360 (Beugeseite des Unterschenkels) und 670 (Brust). Die Zahl am Rücken (782) desselben Materials liegt nahe der der Achselhaut (756) bei meinem gleichaltrigen Material(Fall 2), welchean der Grenze des kleineren Ein- und Zweisechstels der Schwankungsbreite beim Mädchen von K o-

Tabelle 23.

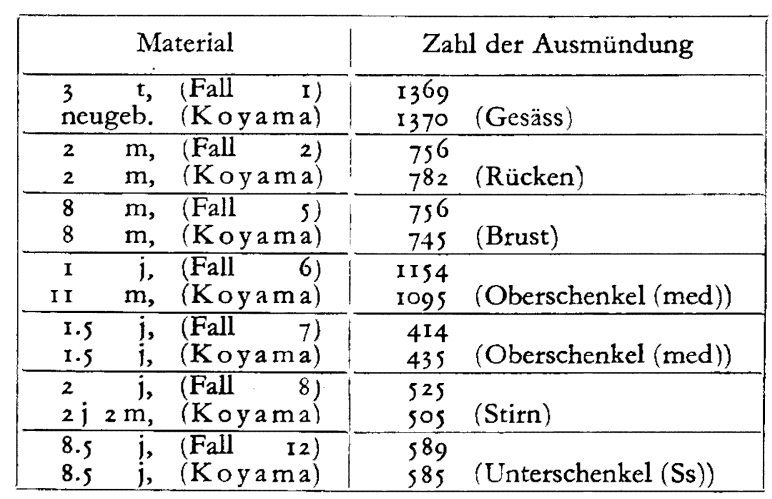

med = Medialseite, $\quad$ Ss $=$ Streckseite.

y a ma sich befindet. Die Ausmündungszahl beim 8 monatigen Kind nach Koy a ma breitet sich zwischen 1435 (Streckseite des Vorderarms) und 540 (Bauch) aus. Die Zahl an der Achselhaut (756) bei meinem gleichaltrigen Material (Fall s) liegt in der Nähe der Zahl an der Brust (754) beim Material von Koy a ma und befindet sich ungefähr an der Grenze des kleineren Zwei- und Einviertels der Schwankungsbreite beim letzteren. Die Ausmündungszahl beim I I monatigen Kind von K o y a ma hat die grösste Zahl an der Streckseite des Unterschenkels (1665) und die geringste Zahl an der Brust (495). Die Zahl ( I I 4) bei meinem fast gleichaltrigen Material (Fall 6, einjähriger) ist beinahe gleich wie die an der Medialseite des Oberschenkels (ro95) des I I monatigen Kindes und liegt etwa an der Grenze des grösseren Vier- und Fünfsiebentels der Schwankungsbreite bei letzteren. Die Zahl beim r.s jährigen Kind von Ko y a ma ist an der Stirn (1280) am grössten und am Bauch (205) am geringsten. Die Zahl an der Achselhaut bei meinem gleichaltrigen Fall 7 ähnelt der an der Medialseite des Oberschenkels (435) bei seinem Material und liegt etwa an der Grenze des kleineren Ein- und Zweifünftels der Schwankungsbreite beim letzteren. Die Zahl beim 2 und $1 / 6$ jährigen Kind von Koy a ma ist am grössten an der Lateralseite des Oberschenkels (I I 83) und am geringsten am Bauch (300). Die Zahl bei meinem 2 jährigen Knaben (Fall 8) (525) entspricht der an der Stirn ( $5 \circ 5)$ bei seinem Material und befindet sich in der Nähe der Grenze des kleineren Ein- und Zweiviertels der Schwankungsbreite beim letzteren. Die Ausmündungszahl beim 8.5 jährigen Kind von Ko y a ma ist am grössten an der Lateralseite des Oberschenkels sowie am Rücken (743), und am geringsten an der Brust (300). Die Zahl bei meinem gleichaltrigen Kind (Fall r 2) (589) ist fast gleich gross wie die an der Streckseite des Unter- 
schenkels ( 585 ) bei seinem Material und liegt fast an der Grenze des grösseren Ein- und Zweidrittels der Schwankungsbreite beim letzteren.

Aus den obigen Befunden kann man wohl schliessen, dass die Ausmündungszahl der e-Drüsen an der Achselhaut im Vergleich mit der an den übrigen verschiedenen Körperteilen des gleichaltrigen Kindes etwas klein ist, mit Ausnahme vom Fall 6 und 2 .

\section{Menge der 'Talgdrüse.}

Das Volumen der Talgdrüsen in I qcm Flächeninhalt der Achselhaut der Kinder ist beim Neugeborenen von 3 Tagen am grössten $(4.606 \mathrm{cmm})$, dann folgen 3 monatiges (1.6I $3 \mathrm{cmm}), 2$ monatiges $(0.857 \mathrm{cmm}), 8$ monatiges $(0.606 \mathrm{cmm}), 8$ jähriges Mädchen $(0.259 \mathrm{cmm}), 3$ monatiger $(0.246 \mathrm{cmm})$, I jähriger $(0.24 \mathrm{I} \mathrm{cmm}), 8.5$ jähriger Knabe $(0.120 \mathrm{cmm})$, I.5 jähriges $(0.099$ $\mathrm{cmm}$ ), 3.5 jähriges Mädchen (0.09I cmm), 2 jähriger Knabe $(0.065 \mathrm{cmm}$ ) und am geringsten ist es beim 4 jährigen Knaben $(0.053 \mathrm{cmm})$, so dass die relative Schwankungsbreite bedeutend gross (ca. 86.8-I) ist (Tab. 24, 25). Wie in der Tabelle 24 und 25 angegeben, nimmt die Talgdrüsenmenge in I qcm Hautstück mit zunehmendem Alter ab, abgesehen vom Fall i I und I 2.

Tabelle 24. Menge der Talgdrüsen und Zahl der Haare in I qcm der Achselhaut der japanischen Kinder.

\begin{tabular}{|c|c|c|c|c|c|c|c|}
\hline Fall & Alter & 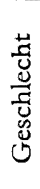 & $\begin{array}{l}\text { Wirkliches } \\
\text { Volumen der } \\
\text { Talgdrüsen } \\
\text { in } \pi \mathrm{r}^{2} \mathrm{qmm} \\
\left(\mathrm{r}=\frac{\mathrm{r} 00}{50} \mathrm{~mm}\right)\end{array}$ & $\begin{array}{l}\text { Volumen } \\
\text { der } \\
\text { Talgdrüsen } \\
\text { in I gcm }\end{array}$ & $\begin{array}{l}\text { Angenom- } \\
\text { mene Aus- } \\
\text { gleichs- } \\
\text { dicke } \\
\text { (rund) }\end{array}$ & $\begin{array}{l}\text { Zahl der } \\
\text { Haare } \\
\text { in I qcm }\end{array}$ & $\begin{array}{l}\text { Angenommenes } \\
\text { Volumen des } \\
\text { Talgdrüsen- } \\
\text { individuums }\end{array}$ \\
\hline I & $\mathbf{t}$ & 우 & $0.578830 \mathrm{cmm}$ & $4.606 \mathrm{cmm}$ & 46. I $u$ & $54 \mathrm{I}$ & $0.008514 \mathrm{cmm}$ \\
\hline 2 & $2 \mathrm{~m}$ & 우 & 0.107028 & 0.852 & 8.5 & 302 & 0.002820 \\
\hline 3 & $\mathrm{~m}$ & 우 & 0.202730 & 1.613 & I6. & 581 & 0.002777 \\
\hline 4 & $\mathrm{~m}$ & 今 & 0.030914 & 0.246 & 2.5 & $27 \mathrm{I}$ & 0.000908 \\
\hline 5 & $\mathrm{~m}$ & q & 0.08 II 31 & 0.646 & 6.5 & 414 & 0.001559 \\
\hline 6 & r j & 今 & 0.030279 & $0.24 \mathrm{I}$ & 2.4 & $6 \times 3$ & 0.000393 \\
\hline 7 & 1.5 & 우 & $0.01239^{\circ}$ & 0.099 & 1.0 & 255 & 0.000387 \\
\hline 8 & 2 & $\delta$ & 0.008183 & 0.065 & 0.7 & 223 & 0.000292 \\
\hline 9 & 3.5 & 우 & 0.011426 & $0.09 \pi$ & 0.9 & 279 & 0.000326 \\
\hline 10 & 4 & $\delta$ & $0.00666 \mathrm{I}$ & 0.053 & 0.5 & 302 & 0.000176 \\
\hline II & 8 & 우 & 0.032547 & 0.259 & 2.6 & 183 & 0.001415 \\
\hline 12 & $8.5 \mathfrak{j}$ & 㑒 & 0.015074 & 0.120 & 1.2 & 326 & 0.000368 \\
\hline
\end{tabular}

Vergleicht man die Befunde von Taniguchi bei einem Neugeborenen und von Min e bei einem 9 monatigen Knaben mit meinen Befunden bei den gleichaltrigen Kindern, so findet man, dass das Volumen der Talgdrüsen in I qcm bei meinem Fall I (Neugeborener) $(4.606 \mathrm{cmm})$ ca. I. 8 mal grösser als das grösste Volumen (Scheitel, 2.610 cmm) der Neugeborenen von Tani- 
Tabelle 25. Reihenfolge der Mengengrösse der Talgdrüsen und der

Zahl der Haare in I qcm der Achselhaut der japanischen Kindern.

\begin{tabular}{|c|c|c|c|c|c|}
\hline \multicolumn{2}{|c|}{$\begin{array}{l}\text { Volumen der Talgdrüsen } \\
\text { in } \mathrm{I} \mathrm{qcm}\end{array}$} & \multicolumn{2}{|c|}{$\begin{array}{c}\text { Volumen des angenommenen } \\
\text { Drüsenindividuums }\end{array}$} & \multicolumn{2}{|c|}{ Zahl der Haare in $\mathrm{I} \mathrm{qcm}$} \\
\hline Fall & Volumen & Fall & Volumen & Fall & Zahl \\
\hline I & $4.606 \mathrm{cmm}$ & $\mathbf{I}$ & $0.008514 \mathrm{cmm}$ & 6 & $6 \times 3$ \\
\hline 3 & 1.613 & 2 & 0.002820 & 3 & $58 \mathrm{I}$ \\
\hline 2 & 0.857 & 3 & 0.002777 & I & 541 \\
\hline 5 & 0.606 & 5 & 0.001559 & 5 & 414 \\
\hline II & 0.259 & IX & 0.001415 & 12 & 326 \\
\hline 4 & 0.246 & 4 & 0.000908 & 10 & 302 \\
\hline 6 & $0.24 \mathrm{I}$ & 6 & 0.000393 & 2 & 302 \\
\hline 12 & 0.120 & 7 & 0.000387 & 9 & 279 \\
\hline 7 & 0.099 & 12 & 0.000368 & 4 & 271 \\
\hline 9 & $0.09 \mathrm{I}$ & 9 & 0.000326 & 7 & 255 \\
\hline 8 & 0.065 & 8 & 0.000292 & 8 & 223 \\
\hline 10 & 0.053 & 10 & 0.000176 & II & 183 \\
\hline
\end{tabular}

g u chi, und das Volumen bei meinem Fall s ( 8 monatiger) $(0.646 \mathrm{cmm}$ ) etwas grösser als das grösste Volumen (Scheitel, $0.598 \mathrm{cmm}$ ) beim 9 monatigen Kind von $M$ in e ist.

Nimmt man nun vorläufig an, dass je eine Talgdrüse zu einem Haare gehört, so wird das angenommene Volumen der einzelnen Talgdrüse leicht berechnet :

\section{Menge der Talgdrüsen in I $\mathrm{qcm}$ \\ Zahl der Haare in $x \mathrm{qcm}$}

Das Volumen dieses angenommenen einzelnen Talgdrüsenindividuums verkleinert sich auch mit zunehmendem Alter, zwei Ausnahmefälle ausgenommen (Fall I I, I 2), indem es beim Fall I am grössten (0.00854 $\mathrm{cmm}$ ) und beim Fall Io am kleinsten $(0.000176 \mathrm{cmm}$ ) (Tab. 24, 25) ist. Die relative Schwankungsbreite ist sehr gross, sich auf ca. 48.4-I belaufend.

Es ist schwer einen bestimmten Zusammenhang zwischen dem Alter und der Haarzahl zu finden, so schwankt die letztere auch nach den Individuen zwischen 6I3 (Fall G) und I83 (Fall I I) (Tab. 25). So beträgt die relative Schwankungsbreite ca. $3 \cdot 3-\mathrm{r}$.

Das Vergleich der Haarzahl in I qcm der Achselhaut mit der der anderen Körperteile bei den fast gleichaltrigen Kindern von Ta nig u chi und Shibay a ma ergibt die Tabelle 26. Nach oben genannten Autoren befindet sich die grösste Haarzahl in I qcm beim 8 monatigen Kind an der Stirn (75 I) und die kleinste an der. Lateralseite des Oberschenkels (107). Die Haarzahl (4I4) bei meinem gleichaltrigen Kind (8 monatigen) liegt derjenigen am Gesäss bei ihrem Material am nächsten und befindet sich etwa in der Mitte der Schwankungsbreite beim letzteren. Die Haarzahl beim i I monatigen Kind von den genannten Autoren ist am grössten an der Stirn (1023) und am kleinsten am Bauch (76). Die Zahl am Scheitel (775) desselben Materials ist etwas 
Tabelle 26.

\begin{tabular}{|c|c|c|c|c|}
\hline \multicolumn{3}{|r|}{ Material } & \multicolumn{2}{|r|}{ Haarzahl } \\
\hline $\begin{array}{l}8 \\
8\end{array}$ & $\mathrm{~m}$ & $\begin{array}{l}\text { (Fall s) } \\
\text { (Taniguchi u. Shibayama) }\end{array}$ & $\begin{array}{l}414 \\
483\end{array}$ & (Gesäss) \\
\hline $\begin{aligned} \text { I } \\
\text { II }\end{aligned}$ & $\mathrm{j}$ & $\begin{array}{l}\text { (Fall 6) } \\
\text { (Taniguchi u. Shibayama) }\end{array}$ & $\begin{array}{l}613 \\
775\end{array}$ & (Scheitel) \\
\hline $\begin{array}{l}3.5 \\
3.5\end{array}$ & $\mathrm{i}$ & $\begin{array}{l}\text { (Fall 9) } \\
\text { (Taniguchi u. Shibayama) }\end{array}$ & $\begin{array}{l}273 \\
221\end{array}$ & (Rücken) \\
\hline $\begin{array}{l}8 \\
7 \cdot 6\end{array}$ & $\mathrm{i}$ & $\begin{array}{l}\text { (Fall II) } \\
\text { (Taniguchi u. Shibayama) }\end{array}$ & $\begin{array}{l}183 \\
155\end{array}$ & (Rücken) \\
\hline
\end{tabular}

grösser als die an der Achselhaut bei meinem Fall 6 (6I3) und die letzte Zahl befindet sich an der Grenze des grösseren Fünf- und Sechsachtels der Schwankungsbreite beim ersteren. Die Haarzahl beim 3.5 jährigen Knaben von Taniguchi und Shibaya ma liegt zwischen 514 an der Stirn und 54 an der Beugeseite des Unterschenkels. Die Zahl (279) bei meinem gleichaltrigen Material (Fall 9) liegt etwa in der Nähe der Zahl am Rücken (221) bei ihrem Material und etwa in der Mitte der Schwankungsbreite beim letzteren. Die Haarzahl beim 7.6 jährigen Knaben von Taniguchi und Shibaya ma ist an der Stirn (549) am grössten und am Bauch (42) am kleinsten. Die Zahl (183) bei meinem 8 jährigen Kind (Fall i I) entspricht der am Rücken (I55) bei ihrem Material und liegt etwa an der Grenze des kleineren Zwei- und Dreisiebentels der Schwankungsbreite beim letzteren.

Aus den obigen Befunden sieht man, dass die Haarzahl an der Achselhaut im Vergleich mit der an den übrigen Körperteilen der gleichaltrigen Kinder fast mittelgross ist.

Das Volumen der zu einer Haargruppe gehörigen Talgdrüse wurde bei den bei jedem Fall absichtlos gewählten Io Haargruppen verfolgt; näheres darüber ist in den Tabellen $27-38$ angegeben.

Das Volumen der einzelnen Talgdrüsen, die mit einer einhaarigen Haargruppe im Zusammenhang stehen, ist beim Fall i I $(0.002465 \mathrm{cmm})$ am grössten und beim Fall $9(0.000153 \mathrm{cmm})$ am kleinsten. Die einzelnen Talgdrüsen, die mit einer aus zwei Haaren bestehenden Haargruppe im Zusammenhang stehen, haben beim Fall I die grösste Menge $(0.019226 \mathrm{cmm})$ und beim Fall Io die kleinste $(0.000230 \mathrm{cmm})$. Die einzelnen Talgdrüsen, die mit einer dreihaarigen Haargruppe im Zusammenhang stehen, haben beim Fall I die grösste Menge $(0.023100 \mathrm{cmm})$ und beim Fall ro die kleinste $(0.000219 \mathrm{cmm})$. Das Volumen der zu einer vierhaarigen Haargruppe gehörigen Drüsen erweist sich beim Fall I als das grösste $(0.03042 \mathrm{Imm})$ und beim Fall I 2 als das kleinste $(0.000307 \mathrm{cmm})$. Die grösste Menge der zu einer fünfhaarigen Haargruppe gehörigen Talgdrüsen ist beim Fall 7 (0.001895 $\mathrm{cmm}$ ) und die kleinste beim Fall $6(0.001490 \mathrm{cmm})$ zu finden. Die sechshaarige Haargruppe 
Tabelle 27-38. Volumen der zu einer Haargruppe gehörigen Talgdrüsen bei den einzelnen Fällen.

Tabelle 27. Fall I.

\begin{tabular}{|c|c|c|}
\hline $\begin{array}{c}\text { Haar- } \\
\text { gruppe }\end{array}$ & $\begin{array}{c}\text { Haarzahl } \\
\text { in einer } \\
\text { Gruppe }\end{array}$ & $\begin{array}{c}\text { Volumen } \\
\text { der Drüsen }\end{array}$ \\
\hline $\mathrm{a}$ & 3 & $0.014789 \mathrm{cmm}$ \\
$\mathrm{b}$ & 3 & 0.024736 \\
$\mathrm{c}$ & 3 & 0.012916 \\
$\mathrm{~d}$ & 2 & 0.019226 \\
$\mathrm{e}$ & 3 & 0.023100 \\
$\mathrm{f}$ & 2 & $0.00621 \mathrm{I}$ \\
$\mathrm{g}$ & 4 & $0.03042 \mathrm{I}$ \\
$\mathrm{h}$ & 3 & 0.022885 \\
$\mathrm{i}$ & 3 & 0.011853 \\
$\mathrm{i}$ & 2 & 0.017473 \\
\hline
\end{tabular}

Tabelle 29. Fall 3.

\begin{tabular}{|c|c|c|}
\hline $\begin{array}{c}\text { Haar- } \\
\text { gruppe }\end{array}$ & $\begin{array}{c}\text { Haarzahl } \\
\text { in einer } \\
\text { Gruppe }\end{array}$ & $\begin{array}{l}\text { Volumen } \\
\text { der Drüsen }\end{array}$ \\
\hline a & 3 & $0.003703 \mathrm{cmm}$ \\
$\mathrm{b}$ & 2 & 0.003560 \\
$\mathrm{c}$ & 3 & $0.01665 \mathrm{I}$ \\
$\mathrm{d}$ & 3 & 0.004765 \\
$\mathrm{e}$ & 4 & 0.006365 \\
$\mathrm{f}$ & 5 & 0.018656 \\
$\mathrm{~g}$ & 2 & $0.01011 \mathrm{II}$ \\
$\mathrm{h}$ & 4 & 0.008665 \\
$\mathrm{i}$ & 3 & 0.006463 \\
$\mathrm{j}$ & 3 & 0.007690 \\
\hline
\end{tabular}

Tabelle 3r. Fall s.

\begin{tabular}{|c|c|c|}
\hline $\begin{array}{c}\text { Haar- } \\
\text { gruppe }\end{array}$ & $\begin{array}{c}\text { Haarzahl } \\
\text { in einer } \\
\text { Gruppe }\end{array}$ & $\begin{array}{c}\text { Volumen } \\
\text { der Drüsen }\end{array}$ \\
\hline $\mathrm{a}$ & 2 & $0.006595 \mathrm{cmm}$ \\
$\mathrm{b}$ & 4 & 0.003615 \\
$\mathrm{c}$ & 3 & 0.007592 \\
$\mathrm{~d}$ & 3 & 0.005182 \\
$\mathrm{e}$ & 2 & 0.003999 \\
$\mathrm{f}$ & 4 & 0.005094 \\
$\mathrm{~g}$ & 3 & 0.006540 \\
$\mathrm{~h}$ & 3 & 0.005565 \\
$\mathrm{i}$ & 2 & 0.003593 \\
$\mathrm{j}$ & 4 & 0.003516 \\
\hline
\end{tabular}

Tabelle 28. Fall 2.

\begin{tabular}{|c|c|c|}
\hline $\begin{array}{c}\text { Haar- } \\
\text { gruppe }\end{array}$ & $\begin{array}{c}\text { Haarzahl } \\
\text { in einer } \\
\text { Gruppe }\end{array}$ & $\begin{array}{l}\text { Volumen } \\
\text { der Drüsen }\end{array}$ \\
\hline $\mathrm{a}$ & 3 & $0.009432 \mathrm{cmm}$ \\
$\mathrm{b}$ & $\mathrm{I}$ & 0.005609 \\
$\mathrm{c}$ & 3 & 0.012269 \\
$\mathrm{~d}$ & 3 & 0.005883 \\
$\mathrm{e}$ & 3 & 0.008676 \\
$\mathrm{f}$ & 4 & 0.010265 \\
$\mathrm{~g}$ & 3 & 0.007997 \\
$\mathrm{~h}$ & 2 & 0.007460 \\
$\mathrm{i}$ & 2 & 0.009859 \\
$\mathrm{j}$ & $\mathrm{I}$ & 0.000263 \\
\hline
\end{tabular}

Tabelle 30. Fall 4.

\begin{tabular}{|c|c|c|}
\hline $\begin{array}{c}\text { Haar- } \\
\text { gruppe }\end{array}$ & $\begin{array}{c}\text { Haarzahl } \\
\text { in einer } \\
\text { Gruppe }\end{array}$ & $\begin{array}{c}\text { Volumen } \\
\text { der Drüsen }\end{array}$ \\
\hline a & 2 & $0.000822 \mathrm{cmm}$ \\
$\mathrm{b}$ & 4 & 0.001326 \\
$\mathrm{c}$ & 3 & 0.001928 \\
$\mathrm{~d}$ & $\mathbf{2}$ & 0.001742 \\
$\mathrm{e}$ & $\mathbf{3}$ & 0.002859 \\
$\mathrm{f}$ & $\mathbf{3}$ & $0.0008 \mathrm{II}$ \\
$\mathrm{g}$ & 4 & 0.001030 \\
$\mathrm{~h}$ & 3 & $0.00265 \mathrm{I}$ \\
$\mathrm{i}$ & 3 & $0.00162 \mathrm{I}$ \\
$\mathrm{j}$ & & 0.001610 \\
\hline
\end{tabular}

Tabelle 32. Fall 6.

\begin{tabular}{|c|c|c|}
\hline $\begin{array}{c}\text { Haar- } \\
\text { gruppe }\end{array}$ & $\begin{array}{c}\text { Haarzahl } \\
\text { in einer } \\
\text { Gruppe }\end{array}$ & $\begin{array}{c}\text { Volumen } \\
\text { der Drüsen }\end{array}$ \\
\hline a & 2 & $0.001983 \mathrm{cmm}$ \\
$\mathrm{b}$ & 4 & 0.000800 \\
$\mathrm{c}$ & 3 & 0.000679 \\
$\mathrm{~d}$ & 2 & 0.001194 \\
$\mathrm{e}$ & 5 & 0.001490 \\
$\mathrm{f}$ & 3 & 0.003254 \\
$\mathrm{~g}$ & 3 & 0.002914 \\
$\mathrm{~h}$ & 5 & 0.001577 \\
$\mathrm{i}$ & 3 & 0.000668 \\
$\mathrm{j}$ & 3 & 0.001983 \\
\hline
\end{tabular}


Quantitative Untersuchung der Anhangsorgane der Achselhaut bei den japanischen Kindern. 44I

Tabelle 33. Fall 7.

\begin{tabular}{|c|c|c|}
\hline $\begin{array}{c}\text { Haar- } \\
\text { gruppe }\end{array}$ & $\begin{array}{c}\text { Haarzahl } \\
\text { in einer } \\
\text { Gruppe }\end{array}$ & $\begin{array}{c}\text { Volumen } \\
\text { der Drüsen }\end{array}$ \\
\hline a & 2 & $0.000942 \mathrm{cmm}$ \\
b & 5 & 0.001895 \\
c & 3 & $0.00093 \mathrm{I}$ \\
d & $\mathbf{2}$ & 0.000646 \\
e & 2 & 0.001008 \\
f & 4 & 0.000898 \\
g & 3 & 0.000679 \\
h & 3 & 0.000745 \\
i & 3 & 0.002 I 47 \\
j & $\mathbf{2}$ & 0.0007 II \\
\hline
\end{tabular}

Tabelle 35. Fall 9.

\begin{tabular}{|c|c|c|}
\hline $\begin{array}{c}\text { Haar- } \\
\text { gruppe }\end{array}$ & $\begin{array}{c}\text { Haarzahl } \\
\text { in einer } \\
\text { Gruppe }\end{array}$ & $\begin{array}{c}\text { Volumen } \\
\text { der Drüsen }\end{array}$ \\
\hline a & I & $\begin{array}{l}0.000153 \mathrm{cmm} \\
\text { b }\end{array}$ \\
c & 3 & 0.001501 \\
d & 3 & 0.001369 \\
e & 3 & 0.001556 \\
f & 3 & 0.000898 \\
g & 3 & 0.001435 \\
h & 3 & 0.001194 \\
i & 3 & 0.000646 \\
j & 2 & 0.000756 \\
\hline
\end{tabular}

Tabelle 37. Fall ir.

\begin{tabular}{|c|c|c|}
\hline $\begin{array}{c}\text { Haar- } \\
\text { gruppe }\end{array}$ & $\begin{array}{c}\text { Haarzahl } \\
\text { in einer } \\
\text { Gruppe }\end{array}$ & $\begin{array}{c}\text { Volumen } \\
\text { der Drüsen }\end{array}$ \\
\hline a & 2 & $0.003166 \mathrm{cmm}$ \\
b & I & 0.002465 \\
c & 3 & 0.006967 \\
d & 2 & 0.002224 \\
e & 2 & 0.000887 \\
f & 2 & 0.003703 \\
g & 2 & 0.001610 \\
h & 2 & 0.005401 \\
i & 2 & 0.002136 \\
j & 2 & 0.002574 \\
\hline
\end{tabular}

fand sich nur selten, und das Volumen der dazu gehörigen Talgdrüsen ist $0.001369 \mathrm{cmm}$.

\begin{tabular}{|c|c|c|}
\hline $\begin{array}{c}\text { Haar- } \\
\text { gruppe }\end{array}$ & $\begin{array}{c}\text { Haarzahl } \\
\text { in einer } \\
\text { Gruppe }\end{array}$ & $\begin{array}{c}\text { Volumen } \\
\text { der Drüsen }\end{array}$ \\
\hline a & 3 & 0.0018 I $8 \mathrm{cmm}$ \\
b & 2 & 0.000679 \\
c & 3 & $0.00058 \mathrm{I}$ \\
d & 3 & 0.000734 \\
e & 2 & 0.001347 \\
f & 2 & 0.000318 \\
g & 2 & 0.001326 \\
h & 2 & 0.000449 \\
i & 2 & 0.001545 \\
j & 4 & 0.000307 \\
\hline
\end{tabular}

Tabelle 34. Fall 8.

\begin{tabular}{|c|c|c|}
\hline $\begin{array}{c}\text { Haar- } \\
\text { gruppe }\end{array}$ & $\begin{array}{c}\text { Haarzahl } \\
\text { in einer } \\
\text { Gruppe }\end{array}$ & $\begin{array}{c}\text { Volumen } \\
\text { der Drüsen }\end{array}$ \\
\hline a & 2 & $0.000767 \mathrm{cmm}$ \\
b & 3 & 0.000515 \\
c & 3 & 0.001271 \\
d & 2 & 0.000504 \\
e & 2 & 0.000340 \\
f & 2 & 0.000438 \\
g & 3 & 0.000372 \\
h & 2 & 0.000131 \\
i & 2 & 0.00128 \\
j & 3 & 0.000887 \\
\hline
\end{tabular}

Tabelle 36. Fall io.

\begin{tabular}{|c|c|c|}
\hline $\begin{array}{c}\text { Haar- } \\
\text { gruppe }\end{array}$ & $\begin{array}{c}\text { Haarzahl } \\
\text { in einer } \\
\text { Gruppe }\end{array}$ & $\begin{array}{c}\text { Volumen } \\
\text { der Drüsen }\end{array}$ \\
\hline $\mathrm{a}$ & 2 & $0.000427 \mathrm{cmm}$ \\
$\mathrm{b}$ & 2 & 0.000460 \\
$\mathrm{c}$ & $\mathrm{I}$ & 0.000767 \\
$\mathrm{~d}$ & 3 & 0.000219 \\
$\mathrm{e}$ & 2 & 0.000909 \\
$\mathrm{f}$ & 3 & 0.000307 \\
$\mathrm{~g}$ & 2 & 0.000230 \\
$\mathrm{~h}$ & $\mathrm{I}$ & 0.000548 \\
$\mathrm{i}$ & 2 & 0.000482 \\
$\mathrm{j}$ & 3 & 0.000964 \\
\hline
\end{tabular}

Tabelle 38. Fall i 2. 


\section{Menge des Haarbalgmuskels.}

Die Menge der Haarbalgmuskeln in I qcm der Achselhaut ('Tab. 39 und 40) ist beim 3 monatigen Mädchen am grössten $\left(0.19^{2} \mathrm{cmm}\right.$ ), dann folgen 8 jähriges (0.191 cmm), 3.5 jähriges Mädchen $(0.094 \mathrm{cmm}), 8.5$ jähriger Knabe $(0.087 \mathrm{cmm}), 3$ monatiges Mädchen $(0.086 \mathrm{cmm}), 2$ jähriger Knabe $(0.086$

Tabelle 39. Menge det Haarbalgmuskeln und Zahl der Haare in I qcm der Achselhaut bei den japanischen Kindern.

\begin{tabular}{|c|c|c|c|c|c|c|c|c|}
\hline Fall & Alte & & 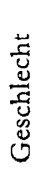 & $\begin{array}{c}\text { Wirkliches } \\
\text { Volumen der } \\
\text { Haarbalgmuskeln } \\
\text { in } \pi^{2} \mathrm{r}^{2} \mathrm{qmm} \\
\left(\mathrm{r}=\frac{100}{50} \mathrm{~mm}\right)\end{array}$ & $\begin{array}{l}\text { Volumen } \\
\text { der } \\
\text { Haarbalg- } \\
\text { muskeln } \\
\text { in I qcm }\end{array}$ & $\begin{array}{l}\text { Angenom- } \\
\text { mene Aus- } \\
\text { gleichs- } \\
\text { dicke } \\
\text { (rund) }\end{array}$ & $\begin{array}{l}\text { Zahl der } \\
\text { Haare } \\
\text { in } 1 \mathrm{qcm}\end{array}$ & $\begin{array}{l}\text { Angenommenes } \\
\text { Volumen des } \\
\text { Muskel- } \\
\text { individuums }\end{array}$ \\
\hline I & 3 & $\mathbf{t}$ & 우 & - & - & - & 541 & - \\
\hline 2 & 2 & $\mathrm{~m}$ & 우 & $0.003845 \mathrm{cmm}$ & $0.03 \mathrm{I} \mathrm{cmm}$ & $0.3 u$ & 302 & $0.000 \mathrm{rol} \mathrm{cmm}$ \\
\hline 3 & 3 & $\mathrm{~m}$ & 우 & 0.010867 & 0.086 & 0.9 & $58 \mathrm{I}$ & 0.000149 \\
\hline 4 & 3 & $\mathrm{~m}$ & ิิ & 0.024177 & 0.192 & 1.9 & 271 & 0.000710 \\
\hline 5 & 8 & $\mathrm{~m}$ & 우 & 0.004897 & 0.039 & 0.4 & $4 \times 4$ & 0.000094 \\
\hline 6 & I & $\mathrm{j}$ & $\hat{\delta}$ & 0.008862 & 0.071 & 0.7 & $6 \times 3$ & 0.000115 \\
\hline 7 & I. 5 & $\hat{j}$ & 우 & 0.010133 & $0.08 \mathrm{I}$ & 0.8 & 255 & 0.000316 \\
\hline 8 & 2 & $\mathfrak{j}$ & $\delta$ & 0.010823 & 0.086 & 0.9 & 223 & 0.000386 \\
\hline 9 & 3.5 & $\mathfrak{j}$ & 우 & 0.011853 & 0.094 & 0.9 & 279 & 0.000338 \\
\hline ro & 4 & i & $\S$ & 0.007153 & 0.057 & 0.6 & 302 & 0000188 \\
\hline I I & 8 & i & 우 & 0.024046 & 0.191 & 1.9 & 183 & 0.001046 \\
\hline I 2 & 8.5 & $\mathrm{i}$ & $\hat{\delta}$ & 0.0109 I I & 0.087 & 0.9 & 326 & 0.000266 \\
\hline
\end{tabular}

Tabelle 40. Reihenfolge der Mengengrösse der Haarbalgmuskeln in I qcm der Achselhaut der japanischen Kinder.

\begin{tabular}{|r|c|c|c|}
\hline \multicolumn{2}{|c|}{$\begin{array}{c}\text { Volumen der Haarbalgmuskeln } \\
\text { in } \mathrm{I} \mathrm{qcm}\end{array}$} & \multicolumn{2}{|c|}{$\begin{array}{c}\text { Volumen des angenommenen } \\
\text { Muskelindividuums }\end{array}$} \\
\hline Fall & Volumen & Fall & Volumen \\
\hline 4 & $0.192 \mathrm{cmm}$ & II & $0.001046 \mathrm{cmm}$ \\
II & 0.191 & 4 & 0.000710 \\
9 & 0.094 & 8 & 0.000386 \\
I & 0.087 & 9 & 0.000338 \\
3 & 0.086 & 7 & 0.000316 \\
8 & 0.086 & 12 & 0.000266 \\
7 & $0.08 \mathrm{I}$ & I0 & 0.000188 \\
6 & $0.07 \mathrm{I}$ & 3 & 0.000148 \\
I0 & 0.057 & 6 & $0.0001 \mathrm{15}$ \\
5 & 0.039 & 2 & 0.000101 \\
2 & $0.03 \mathrm{I}$ & 5 & 0.000094 \\
\hline
\end{tabular}

$\mathrm{cmm})$, I.5 jähriges Mädchen (0.08I cmm), I jähriger $(0.07 \mathrm{I} \mathrm{cmm}), 4$ jähriger Knabe $(0.057 \mathrm{cmm}), 8$ monatiges Mädchen $(0.039 \mathrm{cmm})$, und sie ist am kleinsten beim 2 monatigen Mädchen ( 0.03 I cmm), so dass die relative Schwankungsbreite ca. 6.2-I beträgt. Der Fall I (Neugeborene von 3 Tagen) wurde wegen 
Quantitative Untersuchung der Anhangsorgane der Achselhaut bei den japanischen Kindern. 443

der schlechten Entwicklung des Haarbalgmuskels von dieser Betrachtung ausgenommen.

Vergleicht man das Volumen der Mưskeln in I qcm Hautflächeninhalt bei meinem 8 monatigen Fall $s$ mit dem beim 9 monatigen Knaben von Mine, so findet man das Volumen bei meinem Fall $(0.039 \mathrm{cmm}) \mathrm{sehr} k \mathrm{klein}$, das ca. 1/3.7 des kleinsten Volumens (Beugeseite des Unterschenkels, $0.146 \mathrm{cmm}$ ) bei seinem Material entspricht.

Wenn man vorläufig annimmt, dass je ein Haar einen Arrektormuskel besitzt, so lässt sich das Volumen des angenommenen Muskelindividuums in derselben Weise wie beim angenommenen Talgdrüsenindividuum berechnen :

$$
\frac{\text { Menge der Muskeln in } \mathrm{I} \text { qcm }}{\text { Zahl der Haare in } \mathrm{I} \mathrm{qcm}}
$$

Es ist beim 8 jährigen Mädchen (0.001046 cmm) am grössten und beim 8 monatigen Mädchen (0.000094 cmm) am kleinsten (Tab. 40); also ist die relative Schwankungsbreite ca. I I.I-I.

Tabelle 4I-5 I. Volumen der zu einer Haargruppe gehörigen Haarbalgmuskeln bei den einzelnen Fällen.

Tabelle 4I. Fall 2.

\begin{tabular}{|c|c|c|}
\hline $\begin{array}{c}\text { Haar- } \\
\text { gruppe }\end{array}$ & $\begin{array}{c}\text { Haarzahl } \\
\text { in einer } \\
\text { Gruppe }\end{array}$ & $\begin{array}{c}\text { Volumen der } \\
\text { Nuskeln }\end{array}$ \\
\hline $\mathrm{a}$ & 3 & $0.000208 \mathrm{cmm}$ \\
$\mathrm{b}$ & 3 & 0.000537 \\
$\mathrm{c}$ & 3 & 0.000482 \\
$\mathrm{~d}$ & 3 & 0.000723 \\
$\mathrm{e}$ & 4 & 0.000668 \\
$\mathrm{f}$ & 3 & 0.000460 \\
$\mathrm{~g}$ & 2 & 0.000175 \\
$\mathrm{~h}$ & 3 & 0.000482 \\
$\mathrm{i}$ & 2 & 0.000515 \\
$\mathrm{j}$ & 2 & 0.000405 \\
\hline
\end{tabular}

Tabelle 43. Fall 4.

\begin{tabular}{|c|c|c|}
\hline $\begin{array}{c}\text { Haar- } \\
\text { gruppe }\end{array}$ & $\begin{array}{c}\text { Haarzahl } \\
\text { in einer } \\
\text { Gruppe }\end{array}$ & $\begin{array}{l}\text { Volumen der } \\
\text { Muskeln }\end{array}$ \\
\hline a & 2 & $0.000833 \mathrm{cmm}$ \\
$\mathrm{b}$ & 2 & 0.000668 \\
$\mathrm{c}$ & 3 & 0.002717 \\
$\mathrm{~d}$ & 2 & 0.000833 \\
$\mathrm{e}$ & 3 & 0.000482 \\
$\mathrm{f}$ & 3 & 0.002476 \\
$\mathrm{~g}$ & 2 & 0.000789 \\
$\mathrm{~h}$ & 2 & 0.000329 \\
$\mathrm{i}$ & 4 & 0.002005 \\
$\mathrm{j}$ & 3 & $0.00035 \mathrm{I}$ \\
\hline
\end{tabular}

Tabelle 42. Fall 3.

\begin{tabular}{|c|c|c|}
\hline $\begin{array}{c}\text { Haar- } \\
\text { gruppe }\end{array}$ & $\begin{array}{c}\text { Haarzahl } \\
\text { in einer } \\
\text { Gruppe }\end{array}$ & $\begin{array}{l}\text { Volumen der } \\
\text { Muskeln }\end{array}$ \\
\hline $\mathrm{a}$ & 2 & $0.000679 \mathrm{cmm}$ \\
$\mathrm{b}$ & 4 & 0.000712 \\
$\mathrm{c}$ & 3 & 0.000756 \\
$\mathrm{~d}$ & 2 & 0.000318 \\
$\mathrm{e}$ & 4 & 0.000635 \\
$\mathrm{f}$ & 3 & 0.000548 \\
$\mathrm{~g}$ & 3 & 0.000745 \\
$\mathrm{~h}$ & 3 & 0.001019 \\
$\mathrm{i}$ & 3 & 0.000715 \\
$\mathrm{j}$ & 3 & 0.000646 \\
\hline
\end{tabular}

Tabelle 44. Fall s.

\begin{tabular}{|c|c|c|}
\hline $\begin{array}{c}\text { Haar- } \\
\text { gruppe }\end{array}$ & $\begin{array}{c}\text { Haarzahl } \\
\text { in einer } \\
\text { Gruppe }\end{array}$ & $\begin{array}{c}\text { Volumen der } \\
\text { Muskeln }\end{array}$ \\
\hline a & 3 & $0.000460 \mathrm{cmm}$ \\
b & 2 & 0.000394 \\
c & 3 & 0.000646 \\
d & 4 & 0.001074 \\
e & 4 & 0.000318 \\
f & 3 & 0.000329 \\
g & 3 & 0.000307 \\
h & 2 & 0.000241 \\
i & 3 & 0.000504 \\
i & 4 & 0.000712 \\
\hline
\end{tabular}


Tabelle 45. Fall 6.

\begin{tabular}{|c|c|c|}
\hline $\begin{array}{c}\text { Haar- } \\
\text { gruppe }\end{array}$ & $\begin{array}{c}\text { Haarzahl } \\
\text { in einer } \\
\text { Gruppe }\end{array}$ & $\begin{array}{c}\text { Volumen der } \\
\text { Muskeln }\end{array}$ \\
\hline a & 4 & $0.001030 \mathrm{cmm}$ \\
b & 2 & 0.000340 \\
c & 3 & 0.000537 \\
d & 5 & 0.001369 \\
e & 3 & 0.000340 \\
f & 4 & 0.000767 \\
g & 2 & 0.000252 \\
h & 3 & 0.000515 \\
i & 3 & 0.000208 \\
j & 3 & 0.000285 \\
\hline
\end{tabular}

Tabelle 47. Fall 8.

\begin{tabular}{|c|c|c|}
\hline $\begin{array}{c}\text { Haar- } \\
\text { gruppe }\end{array}$ & $\begin{array}{c}\text { Haarzahl } \\
\text { in einer } \\
\text { Gruppe }\end{array}$ & $\begin{array}{c}\text { Volumen der } \\
\text { Muskeln }\end{array}$ \\
\hline a & 3 & $0.000515 \mathrm{cmm}$ \\
b & 2 & 0.000296 \\
c & 3 & 0.001391 \\
d & 3 & 0.000450 \\
e & 3 & 0.001271 \\
f & 2 & 0.000953 \\
g & 2 & 0.000646 \\
h & 2 & 0.001183 \\
i & 3 & 0.000822 \\
j & 2 & 0.000898 \\
\hline
\end{tabular}

Tabelle 49. Fall Io.

\begin{tabular}{|c|c|c|}
\hline $\begin{array}{c}\text { Haar- } \\
\text { gruppe }\end{array}$ & $\begin{array}{c}\text { Haarzahl } \\
\text { in einer } \\
\text { Gruppe }\end{array}$ & $\begin{array}{c}\text { Volumen der } \\
\text { Muskeln }\end{array}$ \\
\hline a & 2 & $0.000767 \mathrm{cmm}$ \\
$\mathrm{b}$ & 2 & 0.000603 \\
$\mathrm{c}$ & 2 & 0.000603 \\
$\mathrm{~d}$ & 2 & 0.000526 \\
$\mathrm{e}$ & 3 & 0.000613 \\
$\mathrm{f}$ & 2 & 0.000427 \\
$\mathrm{~g}$ & 3 & 0.000559 \\
$\mathrm{~h}$ & 3 & 0.000537 \\
$\mathrm{i}$ & $\mathrm{I}$ & 0.000548 \\
$\mathrm{j}$ & 2 & 0.000603 \\
\hline
\end{tabular}

Tabelle 46. Fall 7.

\begin{tabular}{|c|c|c|}
\hline $\begin{array}{c}\text { Haar- } \\
\text { gruppe }\end{array}$ & $\begin{array}{c}\text { Haarzahl } \\
\text { in einer } \\
\text { Gruppe }\end{array}$ & $\begin{array}{l}\text { Volumen der } \\
\text { Muskeln }\end{array}$ \\
\hline a & 5 & $0.002870 \mathrm{cmm}$ \\
b & 3 & 0.000646 \\
c & 2 & 0.000537 \\
d & 2 & 0.000164 \\
e & 3 & 0.000340 \\
f & 3 & 0.000767 \\
g & 2 & 0.001216 \\
h & 3 & 0.000997 \\
i & 2 & 0.000318 \\
i & 4 & 0.000898 \\
\hline
\end{tabular}

Tabelle 48. Fall 9.

\begin{tabular}{|c|c|c|}
\hline $\begin{array}{c}\text { Haar- } \\
\text { gruppe }\end{array}$ & $\begin{array}{c}\text { Haarzahl } \\
\text { in einer } \\
\text { Gruppe }\end{array}$ & $\begin{array}{c}\text { Volumen der } \\
\text { Muskeln }\end{array}$ \\
\hline a & 3 & $0.001150 \mathrm{cmm}$ \\
$\mathrm{b}$ & 3 & 0.004009 \\
$\mathrm{c}$ & 3 & 0.001063 \\
$\mathrm{~d}$ & 3 & 0.001742 \\
$\mathrm{e}$ & 3 & 0.001643 \\
$\mathrm{f}$ & 3 & 0.001632 \\
$\mathrm{~g}$ & 4 & 0.001227 \\
$\mathrm{~h}$ & 2 & 0.001019 \\
$\mathrm{i}$ & 2 & $0.00081 \mathrm{I}$ \\
$\mathrm{i}$ & 2 & 0.000624 \\
\hline
\end{tabular}

Tabelle so. Fall Ir.

\begin{tabular}{|c|c|c|}
\hline $\begin{array}{c}\text { Haar- } \\
\text { gruppe }\end{array}$ & $\begin{array}{c}\text { Haarzahl } \\
\text { in einer } \\
\text { Gruppe }\end{array}$ & $\begin{array}{l}\text { Volumen der } \\
\text { Muskeln }\end{array}$ \\
\hline a & 2 & $0.003133 \mathrm{cmm}$ \\
b & 2 & 0.000548 \\
c & 2 & 0.002114 \\
d & 2 & 0.004952 \\
e & 2 & 0.001095 \\
f & 5 & 0.000975 \\
g & 3 & 0.000394 \\
h & 2 & 0.001019 \\
i & 2 & 0.003199 \\
j & I & 0.000329 \\
\hline
\end{tabular}


Quantitative Untersuchung der Anhangsorgane der Achselhaut bei den japanischen Kindern. 445

Tabelle 5i. Fall i 2.

\begin{tabular}{|c|c|c|}
\hline $\begin{array}{c}\text { Haar- } \\
\text { gruppe }\end{array}$ & $\begin{array}{c}\text { Haarzahl } \\
\text { in einer } \\
\text { Gruppe }\end{array}$ & $\begin{array}{l}\text { Volumen der } \\
\text { Muskeln }\end{array}$ \\
\hline $\mathrm{a}$ & 2 & $0.000537 \mathrm{cmm}$ \\
$\mathrm{b}$ & 3 & 0.000854 \\
$\mathrm{c}$ & 2 & 0.000449 \\
$\mathrm{~d}$ & 2 & 0.001347 \\
$\mathrm{e}$ & 2 & 0.000241 \\
$\mathrm{f}$ & 3 & 0.001435 \\
$\mathrm{~g}$ & 2 & 0.000164 \\
$\mathrm{~h}$ & 2 & 0.000197 \\
$\mathrm{i}$ & 2 & 0.000142 \\
$\mathrm{j}$ & 2 & 0.000515 \\
\hline
\end{tabular}

Das Volumen des Haarbalgmuskels, welcher mit einer Haargruppe im Zusammenhang steht, wurde bei den bei jedem Fall absichtlos gewählten to Haargruppen untersucht; näheres darüber ist in den Tabellen 4I-SI angegeben.

Das Volumen des zu einer einhaarigen Haargruppe gehörigen Muskels ist beim Fall $100.000548 \mathrm{cmm}$ und beim Fall II $0.000329 \mathrm{cmm}$.

Das Volumen der Muskeln, die mit einer Zweihaargruppe im Zusammenhang stehen, zeigt beim Fall I I den grössten Wert $(0.004952 \mathrm{cmm})$ und beim Fall I 2 den kleinsten $(0.000142 \mathrm{cmm})$. Das Volumen der zu einer Dreihaargruppe gehörigen Muskeln zeigt beim Fall 2 und 6 den kleinsten Wert $(0.000208$ $\mathrm{cmm})$ und beim Fall 9 den grössten $(0.004009 \mathrm{cmm})$. Die grösste Menge der zu einer Vierhaargruppe gehörigen Muskeln ist beim Fall 3 ( 0.002005 $\mathrm{cmm})$ und die kleinste beim Fall $5(0.000318 \mathrm{cmm})$ zu finden. Das Volumen der zu einer Fünfhaargruppe gehörigen Muskeln ist beim Fall 60.001369 $\mathrm{cmm}$ und beim Fall $70.002870 \mathrm{cmm}$.

Tabelle 52. Volumen der Anhangsorgane in I qcm der Achselhaut der japanischen Kinder.

\begin{tabular}{|c|c|c|c|c|}
\hline \multirow{2}{*}{ Fall } & \multicolumn{2}{|c|}{ Schweissdrüsen } & \multirow{2}{*}{ Talgdrüsen } & \multirow{2}{*}{ Haarbalgmuskeln } \\
\hline & a-Drüsen & e-Drüsen & & \\
\hline I & $0.894 \mathrm{cmm}$ & $2.623 \mathrm{cmm}$ & $4.606 \mathrm{cmm}$ & 一 \\
\hline 2 & 0.190 & 1.576 & 0.852 & $0.03 \mathrm{I} \mathrm{cmm}$ \\
\hline 3 & 0.807 & 4. 161 & 1.613 & 0.086 \\
\hline 4 & 0.618 & 3.886 & 0.246 & 0.192 \\
\hline 5 & 2.620 & 4.087 & 0.646 & 0.039 \\
\hline 6 & 0.583 & 3.302 & $0.24 \mathrm{I}$ & $0.07 \mathrm{I}$ \\
\hline 7 & 1.892 & 2.457 & 0.099 & $0.08 \mathrm{I}$ \\
\hline 8 & I.494 & 4.212 & 0.065 & 0.086 \\
\hline 9 & $0.4 \mathrm{II}$ & 3.987 & 0.091 & 0.094 \\
\hline 10 & 1.010 & 3.426 & 0.053 & 0.057 \\
\hline I I & 1.623 & 6.729 & 0.259 & O.I9I \\
\hline 12 & 5.031 & 6.251 & 0.120 & 0.087 \\
\hline $\begin{array}{c}\text { relative } \\
\text { Schwankungs- } \\
\text { breite }\end{array}$ & $26.5-1$ & $4 \cdot 3-1$ & $86.8-1$ & $6.2-1$ \\
\hline
\end{tabular}

Vergleicht man die Menge der oben angeführten, in I qcm der Achselhaut befindlichen, verschiedenen Anhangsorgane bei den einzelnen Fällen miteinander, so bekommt man die Tabelle s2. Beim Fall I ist die Talgdrüsenmenge am grössten, dann folgen die e-Drüsen und a-Drüsen. Beim Fall 2 und 3 ist 
die Menge der e-Drüsen am grössten, dann folgen die Talgdrüsen, a-Drüsen und Muskeln. Beim Fall 4, 5, 6, 7, I I und I 2 ist die Menge der e-Drüsen am grössten, dann folgen die a-Drüsen, Talgdrüsen und Muskeln. Beim Fall 8, 9 und Io ist die Menge der e-Drüsen am grössten, dann folgen die a-Drüsen, Muskeln und Talgdrüsen.

Aus den obigen Befunden kann man wohl schliessen, dass die Reihenfolge der Mengengrösse der in I qcm befindlichen Anhangsorgane der Achselhaut der Kinder in den meisten Fällen wie folgt ist : e-Drüsen, a-Drüsen, Talgdrüsen und Haarbalgmuskeln.

Die relative Schwankungsbreite der Menge der oben angeführten verschiedenen Anhangsorgane in I qcm ist, wie die Tabelle 52 zeigt, bei den Talgdrüsen am grössten, dann folgen die a-Drüsen, Haarbalgmuskeln und am kleinsten ist sie bei den e-Drüsen. Also ist die individuelle Schwankung der Mengengrösse der Anhangsorgane bei den e-Drüsen am kleinsten, und bei den Haarbalgmuskeln ca. 1.4 mal, bei den a-Drüsen ca. 6.2 mal und bei den Talgdrüsen ca. 20.2 mal grösser als bei den ersteren.

\section{Schluss.}

Fasst man die wichtigeren Befunde dieser Arbeit zusammen, so ergibt sich folgendes :

I) Das Volumen sowie die Ausmündungszahl der a-Drüsen in I $\mathrm{qcm}$ Flächeninhalt der Achselhaut und das Volumen des angenommenen a-Drüsenindividuums an derselben Stelle variieren nach dem Alter und individuell erheblich, indem die relative Schwankungsbreite je ca. 26.5-I, 4.3-I und I 1.7-1 beträgt.

Die Ausmündungszahl der a-Drüsen in I qcm der Achselhaut pflegt bei den jüngeren Kindern im grossen und ganzen grösser als bei den älteren zu sein, während das Volumen des angenommenen a-Drüsenindividuums und die a-Drüsenmenge in I $\mathrm{qcm}$ im allgemeinen fast umgekehrt sich verhalten.

Das Volumen des angenommenen a-Drüsenindividuums stimmt mit dem wirklichen durchschnittlichen Volumen der einzelnen a-Drüsen in jedem Fall beinahe überein.

2) Das Volumen sowie die Ausmündungszahl der e-Drüsen in I $\mathrm{qcm}$ Flächeninhalt der Achselhaut und das Volumen des angenommenen e-Drüsenindividuums an derselben Stelle sind nach dem Alter und individuell verschieden gross.

Die relative Schwankungsbreite ist dabei je ca. 4.3-I, 3.3-I, und 5.7-I. Diese relativen Schwankungsbreiten sind alle weit kleiner als dieselben bei den a-Drüsen, was zeigt, dass die individuelle Schwankung des Entwicklungs- 
grades bei den a-Drüsen auffallend grösser als bei den e-Drüsen ist.

Die Menge der e-Drüsen in I qcm und das Volumen des angenommenen e-Drüsenindividuums an der Achselhaut sind bei den älteren Kindern im allgemeinen grösser als bei den jüngeren und bei der Ausmündungszahl der eDrüsen demgegenüber ist es meistens umgekehrt der Fall, obwohl dabei eine ziemlich grosse individuelle Schwankung sich vorfindet.

Die Ausmündungszahl der e-Drüsen in I qcm der Achselhaut ist im Vergleich mit der an den übrigen verschiedenen Körperteilen der gleichaltrigen Kinder meistens verhältnismässig klein.

3) Die Drüsenmenge in I qcm sowie die Ausmündungszahl sind bei allen untersuchten Fällen bei den e-Drüsen grösser als bei den a-Drüsen, während das angenommene Volumen des e-Drüsenindividuums in den meisten Fällen kleiner als das des a-Drüsenindividuums ist.

Bei einem Fall, bei welchem das angenommene Volumen des a-Drüsenindividuums grösser ist, ist auch das des e-Drüsenindividuums groß, während bei der Drüsenmenge und Ausmündungszahl in I qcm diese Verhältnisse kaum zu finden sind.

4) Das Volumen der Talgdrüsen in I qcm der Achselhaut und das des angenommẹnen Talgdrüsenindividuums schwanken nach dem Alter und individuell in sehr hohem Masse und nehmen in den meisten Fällen mit dem Alter ab.

Die relative Schwankungsbreite ist je ca. 86.8-I und 48.4-I.

5) Die Haarzahl an der Achselhaut ist im Vergleich mit der an den anderen Körperteilen der gleichaltrigen Kinder fast mittelgross.

6) Die Menge der Haarbalgmuskeln in I qcm der Achselhaut und das Volumen des angenommenen Muskelindividuums sind auch nach dem Alter und individuell verschieden, wobei die relative Schwankungsbreite je ca. 6.2-I und II.I-I ist.

7) Die Reihenfolge der Mengengrösse der in I qcm befindlichen Anhangsorgane der Achselhaut der Kinder ist meistens wie folgt: e-Drüsen, a-Drüsen, Talgdrüsen und Haarbalgmuskeln.

8) Die individuelle Schwankung der Mengengrösse der Achselhautanhangsorgane in I $\mathrm{qcm}$ ist bei den e-Drüsen am kleinsten, und bei den Haarbalgmuskeln ca. I.4 mal, bei den a-Drüsen ca. 6.2 mal und bei den Talgdrüsen ca. 20.2 mal grösser als bei den ersteren.

Zum Schluss spreche ich Herrn Prof. Dr. T. Taniguchi und Herrn Assistent-Prof. Dr. S. Ka to für ihre stete liebenswürdige Unterstützung bei dieser Arbeit meinen herzlichsten Dank aus. 


\section{Literatur.}

I) Clausen, A., u. A lexanderson, B., Beiträge zur Kenntnis der Entwicklung der Schweissdrüsen des Menschen. Zeitschr. f. mikr.-anat. Forsch. Bd. 30. 1932.

2) Endo, M., Úber die Grösse der apokrinen Schweissdrüsen an der Achselhaut bei den japanischen Kindern. Oka jim as Folia anat. japon. Bd. I7. 1938.

3) Groth, W., Der Verlauf des Drüsenschlauches in den a-Drüsen der Achselhaut des Menschen. Zeitschr. f. mikr.-anat. Forsch. Bd. 38.1935.

4) Herzenberg, H., Neue Beiträge zur Lehre von apokrinen Schweissdrüsen. Virchows Arch. f. path. Anatomie u. Physiologie. Bd. 266. 1927.

s) Hoepke, H., Die Haut: Möllendorffs Handb. d. mikr. Anat. d. Menschen. Bd. III/I. 1927 .

6) Holmgren, E., Die Achseldrüsen des Menschen. Anat. Anz. Bd. 55. I922.

7) Ishikawa, N., Histologische Untersuchungen über die äusseren Genitalien, nebst Achselgrubenhaut und Umgebung des Afters. Acta Scholae Medicinalis Universitatis Imperialis in Kioto. Vol. 6. I923.

8) Ito, T., Quantitative Untersuchung der Anhangsorgane der Haut bei einer japanischen Frau. Folia anat. japon. Bd. 12. 1934.

9) Karrenberg, C. L., Histologische Untersuchungen von Achselorganen bei beiden Geschlechtern. Dermat. Wochenschr. Bd. 87. 1928.

10) Kato, S., Über das Vorkommen apokriner Drüsen in der Aussenhaut des Nasenflügels bei den Japanern. Folia anat. japon. Bd. I4. 1936.

I I) - Untersuchung über die Haar- und Haargruppendichtigkeit bei den japanischen Feten. Folia anat. japon. Bd. x4. 1936.

I2) u. Nagata, M., Kurze Mitteilung über die apokrinen Schweissdrüsen im Vestibulum nasi der Chinesen. Oka j im as Folia anat, japon. Bd. I6. I938.

13) u. U U U U Wer das Vorkommen von Gll. vestibulares nasi bei den Chinesen. Jap. Zeitschr. f. Anat. Bd. I2. 1938. (Ref. Japanisch).

14) u. Minamitani, A., Über das Vorkommen von Gll. alae nasi bei den Chinesen. Jap. Zeitschr. f. Anat. Bd. 12. I938. (Ref. Japanisch).

15) Kawai, M., Quantitative Untersuchung der Anhangsorgane der Haut bei einem erwachsenen Ainu. Folia anat. japon. Bd. Ir. I933.

16) - Quantitative Untersuchung der Anhangsorgane der Haut bei einem japanischen Mädchen. Folia anat. japon. Bd. II. I933.

17) Kawaji, T., Qauntitative Untersuchung der Anhangsorgane der Haut des Handrückens bei den Japanern. Folia anat. japon. Bd. I2. 1934.

18) Keibel, F., u. Mall, F. P., Handb. der Entwicklungsgeschichte des Menschen. Bd. I. igro.

19) Klaar, J., Zur Kenntnis des weiblichen Axillarorgans beim Menschen. Wien. Klin. Wochenschr. Bd. 39. 1926.

20) Koibuchi, S., Quantitative Untersuchung der Anhangsorgane der Haut bei dem japanischen Kind. Folia anat. japon. Bd. Io. 1932.

2r) Kosaka, Y., Quantitative Untersuchung der Anhangsorgane der Haut bei einem japanischen Fetus. Folia anat. japon. Bd. Io. 1932.

22) Koyama, K., Ưber die Verteilung der Schweissdrüsen bei den Japanern. Okajimas Folia anat. japon. Bd. Is. 1937.

23) Kuriki, S., Quantitative Untersuchung der Anhangsorgane der Wangenhaut bei den Japanern. Folia anat. japon. Bd. 13. I935. 
Quantitative Untersuchung der Anhangsorgane der Achselhaut bei den japanischen Kindern. 449

24) Kuriki, S., Quantitative Untersuchung der Schweissdrüse der Handtellerhaut bei den Zwillingen. Folia anat. japon. Bd. 14. 1936.

25) Loeschcke, H., Úber zyklische Vorgänge in den Drüsen des Achselhöhlenorgans und ihre Abhängigkeit vom Sexualzyklus des Weibes. Virchows Arch. f. path. Anatomie u. Physiologie. Bd. 255. I925.

26) Lüneburg, E., Beiträge zur Entwicklung und Histologie der Knäueldrüsen in der Achselhöhle des Menschen. Inaug.-Diss. Rostock. 1902.

27) Okajima, K., u. Kanaizuka, Z., Quantitative Untersuchung des Haarbalgmuskels bei den Säugetieren. Folia anat. japon. Bd. 7. 1929.

28) Mine, T., Quantitative Untersuchung der Anhangsorgane der Haut bei dem japanischen Säugling. Folia anat. japon. Bd. 14. 1936.

29) —_- Über die Grösse der apokrinen Schweissdrüsen an dem Unterbauch bei den Japanerinnen. Okajimas Folia anat. japon, Ergänzungsband zum Bd. I5. I937.

30) Mochizuki, D., Beiträge zur quantitativen Untersuchung der Anhangsorgane der Kopf haut bei einem erwachsenen Hydrokephalus. Folia anat. japon. Bd. r4. 1936.

3I) - Quantitative Untersuchung der Anhangsorgane der Haut bei einpaarigem japanischen eineiigen Zwillingsfoetus. Okaj i m as Folia anat. japon. Bd. I5. 1937.

32) Mogi, E., Beiträge zur Entwicklung der apokrinen Schweissdrüsen im Vestibulum nasi bei den japanischen Feten. Oka ji m as Folia anat. japon. Bd. I6. 1938.

33) Morioka, Y., Beiträge zur Entwicklungsgeschichte der menschlichen embryonalen Axillarschweissdrüsen. Okayama-Igakkai-Zasshi. Jg. 48, Nt. I. 1936. (Japanisch).

34) Nagai, S., Über die Entwicklung der Haare und Schweissdrüsen der oberen Extremitäten. Jap. Zeitschr. f. Anat. Bd. 9. 1936. (Japanisch).

35) Nagata, M., Quantitative Untersuchung der Anhangsorgane der Haut des Nasenflügels bei den Japanern. Oka a i m as Folia anat. japon. Ergänzungsband zum Bd. 15. 1937.

36) Peter, K., Die Gestalt der Achselstoffdrüsen. Zeitschr. f. mikr.-anat. Forsch. Bd. 38. I935.

37) Peter, K., Wetze1, G., u. Heiderich, F., Handb. d. Anat. d. Kindes. Bd. II. 1927.

38) Pinkus, F., Anatomie der Haut. Jadassohns Handb. der Haut- und Geschlechtskrankheiten. Bd. I. 1927.

39) Richter, W., Beiträge zur normalen und pathologischen Anatomie der apokrinen Hautdrüse des Menschen mit besonderer Berücksichtigung des Achselhöhlenorgans. Virchows Arch. f. path. Anat. u. Physiol. Bd. 287. 1933.

40) Schaffer, H., Zur Einteilung der Hautdrüsen. Anat. Anz. Bd. 57. r924.

4r) - Über die Hautdrüsen. Wien. Klin. Wochenschr. Bd. 39. 1926.

42) Shibay ama, H., Quantitative Untersuchung der Anhangsorgane der Haut bei dem BantuXosa. Folia anat. japon. Bd. x3. 1935.

43) —- Quantitative Untersuchung der Anhangsorgane der Kopf haut nebst Bemerkungen über die Haarbalgzahl an den verschiedenen Körperteilen bei einem haarlosęn Neugeborenen (Alopecia congenita). Folia anat. japon. Bd. 13. 1935.

44) Schiefferdecker, P., Die Hautdrüsen des Menschen und der Säugetiere, ihre biologische und rassenanatomische Beobachtung sowie die Muscularis sexualis. Zoologica. H. 72. I922.

45) Sobotta, J., Die Drüse der Haut. Histologie und mikr. Anat. 4. Aufl. r929.

46) Sperling, G., Die Form der apokrinen Hautdrüsen des Menschen. Zeitschr. f. mikr.-anat. Forsch. Bd. 38. 1935.

47) Steiner, K., Die Entwicklung der grossen Schweissdrüsen beim Menschen. Zeitschr. f. Anat. und Entw.-gesch. Bd. $78 . \quad 1926$.

48) St öhr, P., Lehrbuch der Histologie. 22. Aufl. 1930. 
49) Talke, L., Úber die grossen Drüsen der Achselhöhlenhaut des Menschen. Arch. f. mikr. Anat. Bd. 6r. 1903.

50) Taniguchi, T., Quantitative Untersuchung der Anhangsorgane der Haut bei den japanischen Neugeborenen. Folia anat. japon. Bd. 9. I931.

5I) - Kosaka Y., und Nakano, T., Quantitative Untersuchung der Anhangsorgane der Haut bei einem koreanischen Kind. Folia anat. japon. Bd. II. 1933.

52) - und Shibayama, H., Die Dichtigkeit der Körperbehaarung bei den Japanern. Folia anat. japon. Bd. 13. 1935.

53) ——- Quantitative Untersuchung der Ảnhangsorgane der Haut bei einem Hydrokephalus. Folia anat. japon. Bd. 13. 1935.

54) —, Úber die Verteilung der Schweissdrüsen bei Menschen. Nagoya Iji Shinshi. Bd. 8. 1934. (Japanisch).

55) - - und Mochizuki D., Über die Verteilung der Schweissdrüsen bei den japanischen Feten. Okajimas Folia anat. japon. Ergänzungsband zum Bd. Is. 1937.

56) Waelsch, L., Veränderung der Achselschweissdrüsen während der Schwangerschaft. Münch. Med. Wochenschr. Jg. 59. 1912.

57) — Über Veränderung der Achselschweissdrüsen während der Gravidität. Arch. f. Derm. u. Syph. Bd. II4. I912. 